\title{
Development of a Perfectly Matched Layer Technique for a Discontinuous-Galerkin Spectral-Element Method
}

\author{
Anirban Garai*, Laslo T. Diosady ${ }^{\dagger}$, Scott M. Murman ${ }^{\ddagger}$, and Nateri K. Madavan ${ }^{\S}$ \\ NASA Ames Research Center, Moffett Field, CA, USA
}

\begin{abstract}
The perfectly matched layer (PML) technique is developed in the context of a highorder spectral-element Discontinuous-Galerkin (DG) method. The technique is applied to a range of test cases and is shown to be superior compared to other approaches, such as those based on using characteristic boundary conditions and sponge layers, for treating the inflow and outflow boundaries of computational domains. In general, the PML technique improves the quality of the numerical results for simulations of practical flow configurations, but it also exhibits some instabilities for large perturbations. A preliminary analysis that attempts to understand the source of these instabilities is discussed.
\end{abstract}

\section{Introduction}

The numerical simulation of unsteady aerodynamic flows of practical interest involves discretized computational domains that often must be artificially truncated. Appropriate boundary conditions are required at these truncated domain boundaries. Ideally, these boundary conditions should be perfectly "absorbing" or "nonreflecting" to ensure that they do not contaminate the flow field in the interior of the domain. The proper specification of these boundaries is critical to the stability, accuracy, convergence, and quality of the numerical solution, ${ }^{1}$ and has been the topic of considerable research. The need for such accurate boundary specification has been underscored in recent years stemming from increased interest in applying higher-fidelity methods (DNS, LES, etc.) in conjunction with high-order low-dissipation numerical schemes to realistic flow configurations.

One of the most popular choices for specifying these boundaries is to use characteristic-based boundary conditions $^{2-7}$ where the linearized flow field at the boundaries is decomposed into characteristic waves using either one-dimensional Riemann ${ }^{2-5}$ or other multi-dimensional Riemann approximations. ${ }^{6,7}$ The boundary conditions are set such that the outgoing characteristics are taken from the interior of the domain, while only the incoming characteristics into the domain are specified by the boundary conditions. For unsteady simulations, time-dependent boundary data may not be available; however, not specifying any incoming characteristics makes the problem ill-posed. In such situations, an approach using an ad-hoc parameter to force the solution back to some mean state renders the solution stable while allowing small reflections. ${ }^{5}$ Generally speaking, characteristic conditions work reasonably well when the flow direction is normal to the boundary, but reflect spurious energy otherwise.

An alternative to characteristic-based boundary conditions is to add additional "buffer" regions that augment the main computational domain near the artificial boundaries, and solve different sets of equations in the buffer regions in order to minimize acoustic reflections. One approach involves modeling the pressure fluctuations as acoustic waves propagating in the far-field relative to a single noise source inside the buffer region. ${ }^{8-10}$ This approach treats the vorticity-induced pressure fluctuations in the same manner as the acoustic waves. Another popular approach, often referred to as the "sponge layer," attempts to dampen

\footnotetext{
*Postdoctoral Fellow, Oak Ridge Associated Universities, Anirban.Garai@nasa.gov

†Science and Technology Corporation, Laslo.Diosady@nasa.gov

${ }^{\ddagger}$ Computational Physics Branch, NASA Advanced Supercomputing Division. Scott.M.Murman@nasa.gov

$\S$ Computational Aerosciences Branch, NASA Advanced Supercomputing Division. Nateri.K.Madavan@nasa.gov
} 
the flow perturbations by introducing artificial dissipation in the buffer region. ${ }^{11}$ Although the artificial dissipation removes all perturbations inside the sponge layer, incoming waves are still reflected from the interface boundary between the computational domain and the sponge layer. The effect of these reflections can be somewhat mitigated by appropriately selecting the strength of the artificial dissipation and the physical extent of the sponge layer.

One of the most promising variants of the buffer region approach is the Perfectly Matched Layer (PML) technique. The PML technique mitigates spurious reflections from the boundaries and interfaces by damping the perturbation modes inside the buffer region such that their eigenfunctions remain unchanged. The technique was first developed by Berenger ${ }^{12}$ for application to problems involving electromagnetic wave propagation. It was later extended to the linearized Euler, Euler, and Navier-Stokes equations by Hu and his coauthors. ${ }^{13-19}$ The PML technique ensures the no-reflection property for all waves, irrespective of incidence angle, wavelength, and propagation direction. Although the technique requires the solution of a set of auxiliary equations, the computational overhead can be justified since it allows use of smaller domain sizes and can provide better accuracy, and convergence of the numerical solution.

In this paper, the PML technique is developed in the context of a high-order spectral-element Discontinuous-Galerkin (DG) method. The technique is compared to other approaches, such as those based on using characteristic boundary conditions and sponge layers, for treating the inflow and outflow boundaries of computational domains. The superiority of the current PML technique is demonstrated for a range of test cases, viz., acoustic pulse propagation, convective vortex, shear layer flow, low-pressure turbine cascade flow, and flow over a bluff body. The paper is structured as follows. The PML equations are first derived from the Navier-Stokes equations, followed by a brief description of the higher-order DG method used here. Results for the test cases considered are then presented, and success and failure modes of the PML technique are identified. The nonlinear instabilities arising in the PML domain for large perturbations are then addressed, followed by some concluding remarks.

\section{Theory}

We first consider the derivation of the one-dimensional (without loss of any generality) $x_{1}$-layer PML equations using the conservative form of the compressible nonlinear Navier-Stokes equations:

$$
\boldsymbol{u}_{, t}+\boldsymbol{F}_{i, x_{i}}=0,
$$

where $\boldsymbol{u}=\left\{\rho, \rho u_{j}, \rho E\right\}$ are the conservative variables, and $\boldsymbol{F}_{i}$ is the total flux. Here, $\rho, u_{j}, E=\frac{p}{(\gamma-1) \rho}+$

$\frac{1}{2} u_{j} u_{j}, p$, and $\gamma$ are the density, velocity, total energy, pressure, and specific heat ratio, respectively. The conservative state, $\boldsymbol{u}$, can be partitioned into a mean state, $\overline{\boldsymbol{u}}$, and a perturbation, $\boldsymbol{u}^{\prime}$. The mean state, $\overline{\boldsymbol{u}}$, is a solution of the steady Navier-Stokes equations and is hereafter referred to as the target state. It follows from the definition of $\overline{\boldsymbol{u}}$ that:

$$
\overline{\boldsymbol{F}}_{i, x_{i}}=0,
$$

where $\overline{\boldsymbol{F}_{i}}=\boldsymbol{F}_{i}(\overline{\boldsymbol{u}})$. Note that the equality in equation (2) can be relaxed with minimal effect on the final solution. ${ }^{17,18}$ The equations for $\boldsymbol{u}^{\prime}$ can then be derived by subtracting equation (2) from equation (1):

$$
\boldsymbol{u}_{, t}^{\prime}+\left(\boldsymbol{F}_{i}-\overline{\boldsymbol{F}_{i}}\right)_{, x_{i}}=0 .
$$

Following Berenger ${ }^{12}$ and $\mathrm{Hu},{ }^{13,14}$ the $\boldsymbol{u}^{\prime}$ variables are split as $\boldsymbol{u}^{\prime}=\boldsymbol{u}_{1}^{\prime}+\boldsymbol{u}_{2}^{\prime}+\boldsymbol{u}_{3}^{\prime}$ to rewrite equation (3) as:

$$
\begin{gathered}
\boldsymbol{u}_{1, t}^{\prime}+\left(\boldsymbol{F}_{1}-\overline{\boldsymbol{F}_{1}}\right)_{x_{1}}=0, \\
\boldsymbol{u}_{2, t}^{\prime}+\left(\boldsymbol{F}_{2}-\overline{\boldsymbol{F}_{2}}\right)_{x_{2}}=0, \\
\boldsymbol{u}_{3, t}^{\prime}+\left(\boldsymbol{F}_{3}-\overline{\boldsymbol{F}_{3}}\right)_{x_{3}}=0 .
\end{gathered}
$$

Performing a Fourier transform on equation (4) yields:

$$
\begin{aligned}
& (-i \omega) \widetilde{\boldsymbol{u}_{1}^{\prime}}+\left(\widetilde{\boldsymbol{F}_{1}}-\widetilde{\boldsymbol{F}_{1}}\right)_{, x_{1}}=0, \\
& (-i \omega) \widetilde{\boldsymbol{u}_{2}^{\prime}}+\left(\widetilde{\boldsymbol{F}_{2}}-\widetilde{\boldsymbol{F}_{2}}\right)_{, x_{2}}=0,
\end{aligned}
$$




$$
(-i \omega) \widetilde{\boldsymbol{u}_{3}^{\prime}}+\left(\widetilde{\boldsymbol{F}_{3}}-\widetilde{\overline{\boldsymbol{F}_{3}}}\right)_{x_{3}}=0
$$

where $\widetilde{\boldsymbol{u}}$ is the Fourier transform of $(\boldsymbol{u})$. In order to apply the PML layer only in the $x_{1}$ direction, the spatial derivative $\frac{1}{\partial x_{1}}$ is transformed to $\frac{1}{\left(1+\frac{i \sigma}{\omega}\right) \partial \breve{x}_{1}}$, where $\sigma$ is the absorption coefficient. To ensure that there are no reflections at the interface, $\sigma$ can vary only in the $x_{1}$ direction for the $x_{1}$ PML layer. With the transformation, equation (5) is rewritten as (with the accents on the independent variables removed for convenience):

$$
\begin{gathered}
(-i \omega)\left(1+\frac{i \sigma}{\omega}\right) \widetilde{\boldsymbol{u}_{1}^{\prime}}+\left(\widetilde{\boldsymbol{F}_{1}}-\widetilde{\overline{\boldsymbol{F}_{1}}}\right)_{x_{1}}=0, \\
(-i \omega) \widetilde{\boldsymbol{u}_{2}^{\prime}}+\left(\widetilde{\boldsymbol{F}_{2}}-\widetilde{\widetilde{\boldsymbol{F}_{2}}}\right)_{x_{2}}=0, \\
(-i \omega) \widetilde{\boldsymbol{u}_{3}^{\prime}}+\left(\widetilde{\boldsymbol{F}_{3}}-\widetilde{\overline{\boldsymbol{F}_{3}}}\right)_{, x_{3}}=0 .
\end{gathered}
$$

Defining an auxiliary variable $\boldsymbol{Q}$ as $\boldsymbol{u}_{1}^{\prime}$, and adding the equations (6a-c), the "split" form of the PML equations for the perturbed variables $\boldsymbol{u}^{\prime}$ is:

$$
\begin{aligned}
& (-i \omega) \widetilde{\boldsymbol{u}^{\prime}}+\left(\widetilde{\boldsymbol{F}_{i}}-\widetilde{\overline{\boldsymbol{F}_{i}}}\right)_{, x_{i}}+\sigma \widetilde{\boldsymbol{Q}}=0, \\
& (-i \omega) \widetilde{\boldsymbol{Q}}+\left(\widetilde{\boldsymbol{F}_{1}}-\widetilde{\overline{\boldsymbol{F}_{1}}}\right)_{, x_{1}}+\sigma \widetilde{\boldsymbol{Q}}=0 .
\end{aligned}
$$

Reverting equation (7) back to the physical coordinate system, and adding equation (7a) to equation (2), the Navier-Stokes PML equations for the $x_{1}$-layer are:

$$
\begin{gathered}
\boldsymbol{u}_{, t}+\boldsymbol{F}_{i, x_{i}}+\sigma \boldsymbol{Q}=0, \\
\boldsymbol{Q}_{, t}+\left(\boldsymbol{F}_{1}-\overline{\boldsymbol{F}_{1}}\right)_{, x_{1}}+\sigma \boldsymbol{Q}=0 .
\end{gathered}
$$

It is important to note that the PML equations (8) do not reflect any waves at the interface for electromagnetics, ${ }^{12}$ and linear Euler problems ${ }^{13}$ for the continuous equations. However, Hu ${ }^{13}$ noted very small reflections from the interface for the discretized equations.

$\mathrm{Hu}^{14}$ also showed that the PML equations (8) may become unstable for certain perturbations, even for the linear Euler case with constant mean flow. This instability arises due to the misalignment of the group and phase velocities of the acoustic waves. ${ }^{14,16,17}$ By applying a variable transformation: $\breve{t} \rightarrow t+\beta x_{1}$, $\breve{x}_{i} \rightarrow x_{i}$, where $\beta=\frac{u}{c^{2}-u^{2}}$ and $c=\sqrt{\gamma p / \rho}$ is the speed of sound, the group and phase velocities become perfectly aligned for the linearized Euler case with uniform flow. However, it is difficult to determine the dispersion relation in the case of more general flows, and $\mathrm{Hu}^{17}$ reported that $\beta \approx \frac{u_{b}}{c_{b}^{2}-u_{b}^{2}}$, where $u_{b}$, $c_{b}$ are the bulk velocity and speed of sound, is a good approximation. However, $\mathrm{Hu}^{17}$ also observed that this correction can become unstable for large $\sigma$. With this given transformation, equations (8) become (with the accents on the independent variables removed for convenience):

$$
\begin{gathered}
\boldsymbol{u}_{, t}+\boldsymbol{F}_{i, x_{i}}+\sigma \beta\left(\boldsymbol{F}_{1}-\overline{\boldsymbol{F}_{1}}\right)+\sigma \boldsymbol{Q}=0, \\
\boldsymbol{Q}_{, t}+\left(\boldsymbol{F}_{1}-\overline{\boldsymbol{F}_{1}}\right)_{, x_{1}}+\sigma \beta\left(\boldsymbol{F}_{1}-\overline{\boldsymbol{F}_{1}}\right)+\sigma \boldsymbol{Q}=0,
\end{gathered}
$$

The PML equations (9) also become unstable in the presence of crossflow, ${ }^{20}$ since the group and phase velocities become misaligned for vorticity and entropy waves. Using $\breve{x}_{1} \rightarrow x_{1}, \breve{x}_{2} \rightarrow x_{2}-V_{o} t, \breve{x}_{3} \rightarrow x_{3}-W_{o} t$, and $\breve{t} \rightarrow t$, where $V_{o}$ and $W_{o}$ are the bulk velocities in $x_{2}$ and $x_{3}$ directions respectively, improves the stability of PML equations (9) in the presence of crossflow. Applying this transformation, equations (9) become (again, without the accents on the independent variables):

$$
\begin{gathered}
\boldsymbol{u}_{, t}+\boldsymbol{F}_{i, x_{i}}+\sigma \beta\left(\boldsymbol{F}_{1}-\overline{\boldsymbol{F}_{1}}\right)+\sigma \boldsymbol{Q}=0 \\
\boldsymbol{Q}_{, t}+\left(\boldsymbol{F}_{1}-\overline{\boldsymbol{F}_{1}}\right)_{x_{1}}+V_{0} \boldsymbol{Q}_{x_{2}}+W_{0} \boldsymbol{Q}_{x_{3}}+\sigma \beta\left(\boldsymbol{F}_{1}-\overline{\boldsymbol{F}_{1}}\right)+\sigma \boldsymbol{Q}=0,
\end{gathered}
$$

Note the final form of the PML equations (10) are fully nonlinear.

Although the PML equations (10) are derived for the Navier-Stokes equations, we have omitted the viscous flux contribution. Strictly speaking, one has to solve another set of auxiliary equations in order to apply the PML technique to the Navier-Stokes equations. ${ }^{18}$ For now, we do not include any additional equations. 
We note that one major issue with regard to implementing any PML approach for subsonic flows is in the specification of the target state. At inflow boundaries, the reservoir state is generally known, but at the outflow boundaries the state values are not all known a priori. The complete state needs to be inferred and specified at the outflow boundaries in order to employ the buffer region approach, and the accuracy of the final solution will depends on the quality of this assumption. The approach used here is to conduct a precursor simulation without any PML, and to use the mean solution from this simulation as the target state for successive simulations incorporating the PML technique.

\section{Numerical method}

The spectral-element DG solver used in this paper is described in this section. The spatial domain, $\Omega$, is partitioned into non-overlapping hexahedral elements, $\kappa$, while the time is partitioned into time intervals (or time-slabs), $I^{n}=\left[t^{n}, t^{n+1}\right]$. After defining $\mathcal{V}_{h}=\left\{\boldsymbol{w},\left.\boldsymbol{w}\right|_{\kappa} \in[\mathcal{P}(\kappa \times I)]^{5}\right\}$, the space-time finite-element space is discretized using piece-wise polynomial functions in both space and time. The governing equations (10) are written in weak form as:

$$
\begin{gathered}
\sum_{\kappa}\left\{\int_{I} \int_{\kappa}\left[-\left(\boldsymbol{w}_{, t} \boldsymbol{u}+\boldsymbol{w}_{x_{i}} \boldsymbol{F}_{i}\right)+\boldsymbol{w}\left(\sigma \beta\left(\boldsymbol{F}_{1}-\overline{\boldsymbol{F}_{1}}\right)+\sigma \boldsymbol{Q}\right)\right]\right. \\
\left.+\int_{I} \int_{\partial \kappa} \boldsymbol{w}\left(\widehat{\boldsymbol{F}}_{i}^{\boldsymbol{n}_{i}}+\widehat{\boldsymbol{F}}_{i}^{V} \boldsymbol{n}_{i}\right)+\int_{\kappa}\left[\boldsymbol{w}\left(t_{-}^{n+1}\right) \boldsymbol{u}\left(t_{-}^{n+1}\right)-\boldsymbol{w}\left(t_{+}^{n}\right) \boldsymbol{u}\left(t_{-}^{n}\right)\right]\right\}=0, \\
\sum_{\kappa}\left\{\int_{I} \int_{\kappa}\left[-\left(\boldsymbol{w}_{, t} \boldsymbol{Q}+\boldsymbol{w}_{x_{1}}\left(\boldsymbol{F}_{1}-\overline{\boldsymbol{F}_{1}}\right)+\boldsymbol{w}_{x_{2}} V_{0} \boldsymbol{Q}+\boldsymbol{w}_{x_{3}} W_{0} \boldsymbol{Q}\right)+\boldsymbol{w}\left(\sigma \beta\left(\boldsymbol{F}_{1}-\overline{\boldsymbol{F}_{1}}\right)+\sigma \boldsymbol{Q}\right)\right]\right. \\
+\int_{I} \int_{\partial \kappa} \boldsymbol{w}\left(\widehat{\boldsymbol{F}_{1}^{I} \boldsymbol{n}_{1}}-\widehat{\boldsymbol{F}_{1}^{I} \boldsymbol{n}_{1}}+\widehat{\boldsymbol{F}_{1}^{V} \boldsymbol{n}_{1}}-\widehat{\boldsymbol{F}}_{1}^{V} \boldsymbol{n}_{1}\right) \\
\left.+\boldsymbol{w}\left(V_{0} n_{1}+W_{0} n_{2}\right) \widehat{\boldsymbol{Q}}+\int_{\kappa}\left[\boldsymbol{w}\left(t_{-}^{n+1}\right) \boldsymbol{Q}\left(t_{-}^{n+1}\right)-\boldsymbol{w}\left(t_{+}^{n}\right) \boldsymbol{Q}\left(t_{-}^{n}\right)\right]\right\}=0,
\end{gathered}
$$

where the second and third integrals arise due to the spatial and temporal discontinuity of the basis functions, respectively. Here, $\widehat{\boldsymbol{F}}_{i}^{\boldsymbol{I}} \boldsymbol{n}_{i}, \widehat{\boldsymbol{F}}_{i}^{V} \boldsymbol{n}_{i}$, and $\left(V_{0} n_{1}+W_{0} n_{2}\right) \widehat{\boldsymbol{Q}}$ denote single-valued numerical flux functions approximating the inviscid, viscous and $\boldsymbol{Q}$ fluxes at the spatial boundaries of the elements, respectively. In this work, the inviscid flux is computed using the Ismail and Roe flux, ${ }^{21}$ the viscous flux is computed using the method of Bassi and Rebay, ${ }^{22}$ and the $\boldsymbol{Q}$ fluxes are computed using a fully upwind method. We use an entropy variable formulation, where we seek a solution of the form $\boldsymbol{u}=\boldsymbol{u}(\boldsymbol{v})$, where $\boldsymbol{v}=\left\{\frac{s}{\gamma-1}+\frac{\gamma+1}{\gamma-1}-\frac{\rho E}{p}, \frac{\rho u_{j}}{p},-\frac{\rho}{p}\right\}$ is the vector of entropy variables and $s\left(=\log \left(p / \rho^{\gamma}\right)\right)$ is the entropy. We seek a solution $(\boldsymbol{v}, \boldsymbol{Q})$ with $\boldsymbol{v}, \boldsymbol{Q} \in \mathcal{V}_{h}$ that satisfies the weak form equation (11) for all $\boldsymbol{w} \in \mathcal{V}_{h}$. The space $\mathcal{V}_{h}$ is spanned by the tensor product of 1D nodal Lagrange basis functions defined at the Gauss-Legendre points. Integrals in equation (11) are approximated with a quadrature rule using twice the number of quadrature points as solution points in each coordinate direction in order to minimize quadrature errors. The resulting nonlinear system of equations is then solved using a preconditioned Jacobian-free Newton-Krylov solver. ${ }^{23,24}$

For simulations that incorporate buffer regions, there are two adjustable parameters, the absorption coefficient, $\sigma$, and the buffer width. These parameters control the reflections from the interfaces and boundaries for the sponge layer cases, and the reflections from the boundaries for the PML cases. For the PML technique, the stability of the numerical simulation is also a function of $\sigma$. One expects that $\boldsymbol{Q}$ will be zero at a boundary since the flow solution here should match the target state, and will vary at the interface depending on the incoming disturbances from the domain of interest. Thus, non-zero values for $\boldsymbol{Q}$ at a boundary are an indication that the absorption coefficient and/or buffer width for the PML may be too small. The absorption coefficient, $\sigma$, is set to zero in the domain of interest for the sponge layer and PML simulations. Normally $\sigma$ is increased from a value of zero at the interface to a desired value inside the buffer layer using some power law for finite-difference and finite-volume solvers. However, since discontinuities can be handled efficiently in the DG approach, a constant value for $\sigma$ is used in the buffer layers in the present study. ${ }^{15}$ 


\section{Computational results}

In order to assess and demonstrate its capabilities the PML technique was applied to the following five test cases:
A. Propagation of an acoustic pulse in an inviscid fluid,
B. Convection of an isentropic vortex,
C. Two-dimensional shear flow,
D. Flow through a low-pressure turbine cascade,
E. Flow over a bluff body (block in this case).

The flow physics in test case A is primarily acoustic in nature, in test case B is vortical, while in test case C it is influenced by both vorticity and acoustics. These three test cases have been extensively studied by $\mathrm{Hu}$ and his coauthors. ${ }^{13-19}$ Rest of the test cases, D and E, demonstrate the potential of the PML technique when applied to realistic flow problems. For all test cases results obtained using the PML technique are compared to two other popular boundary treatment techniques: characteristic boundary conditions without any buffer regions, ${ }^{1,5}$ and a sponge layer approach ${ }^{1}$ in the $x_{1}$ direction. All the solutions presented here are obtained using a spatial 8th-order and temporal 4th-order DG scheme. For the remainder of the paper, characteristic boundary condition simulations will refer to simulations without any buffer regions. For these simulations, the computational domain comprises only the domain of interest, whereas for the sponge and PML simulations buffer regions were added on both sides of the domain of interest (Figure 1) and the same characteristic boundary conditions were used at the buffer region boundaries (hereafter referred as boundaries). In order to quantify the behavior and performance of the three different artificial boundary treatments, additional simulations with extended domains were also performed for the first two test cases, since the analytical solutions are available for these cases; these are referred to as infinite domain simulations.

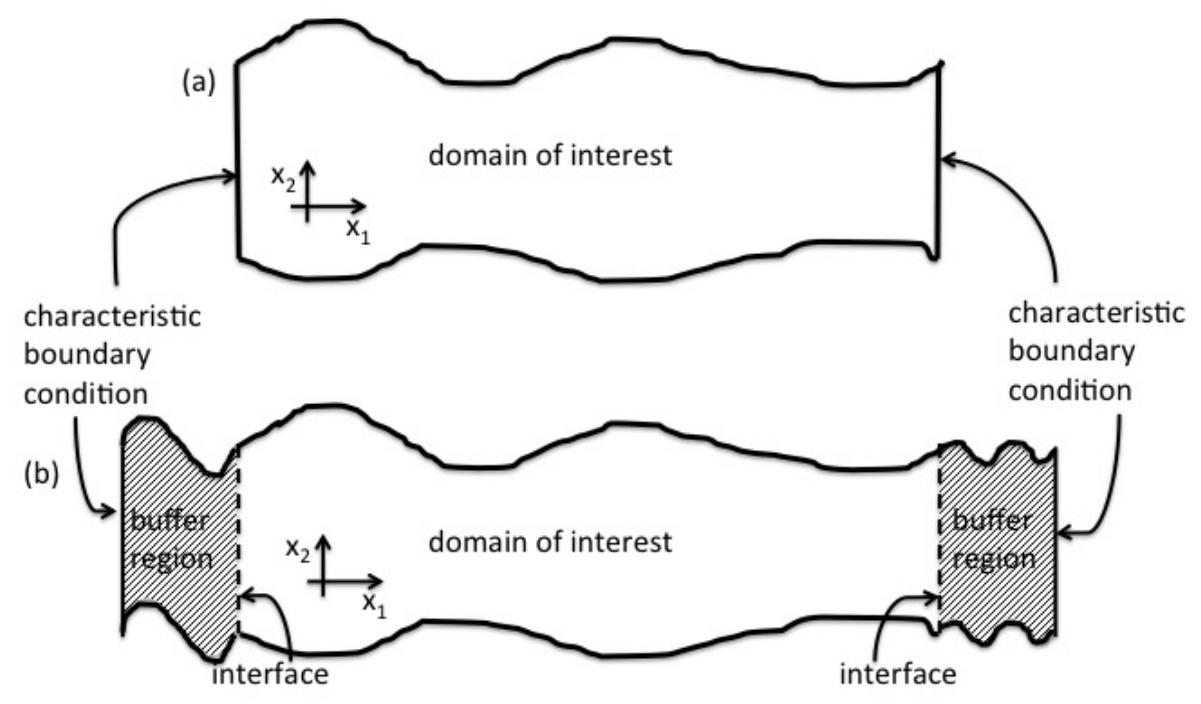

Figure 1. Schematic of the computational domains for simulations with (a) characteristic, and (b) sponge and PML boundary conditions. 
The sponge layer technique can be simulated using equations (10), by including only $\sigma(\boldsymbol{u}-\overline{\boldsymbol{u}})$ in equations (10a), without having to solve any equations (10b) for the auxiliary variables $\boldsymbol{Q}$. The auxiliary variables $\boldsymbol{Q}$ were initialized to zero for the PML layer simulations. Boundary conditions (both at the boundary and the interface) for the $\boldsymbol{Q}$ variable in the $x_{1}$ direction for the $x_{1}$-PML layer are not needed (refer to equations $(10 \mathrm{~b}))$.

\section{A. Acoustic pulse}

An acoustic pulse in an inviscid fluid was simulated in the domain, $-3 \leq x_{1} \leq 3 ;-7.5 \leq x_{2} \leq 7.5$, using 12 and 30 elements in the $x_{1}$ and $x_{2}$ directions, respectively. The initial condition was prescribed as: $\rho_{\infty}$, $u_{j \infty}=0, p=p_{\infty}+\frac{p_{\infty}}{1000} \exp ^{-25 \log (2)\left(x_{1}^{2}+x_{2}^{2}\right)}$. Far-field and periodic boundary conditions were used in the $x_{2}$ and $x_{3}$ directions, respectively. For simulations with buffer regions, two domains of unit width were added to the left of $x_{1}=-3$ and to the right of $x_{1}=3$ using 2 elements in the $x_{1}$ direction. The target states in the buffer region were constructed using $\rho_{\infty}, u_{j \infty}$, and $p_{\infty}$. The absorption coefficient was chosen as $\sigma=1$ for both the sponge layer and PML simulations. The parameters $\beta, V_{0}$, and $W_{0}$ were set to zero as there was no flow in this case for the PML simulations.

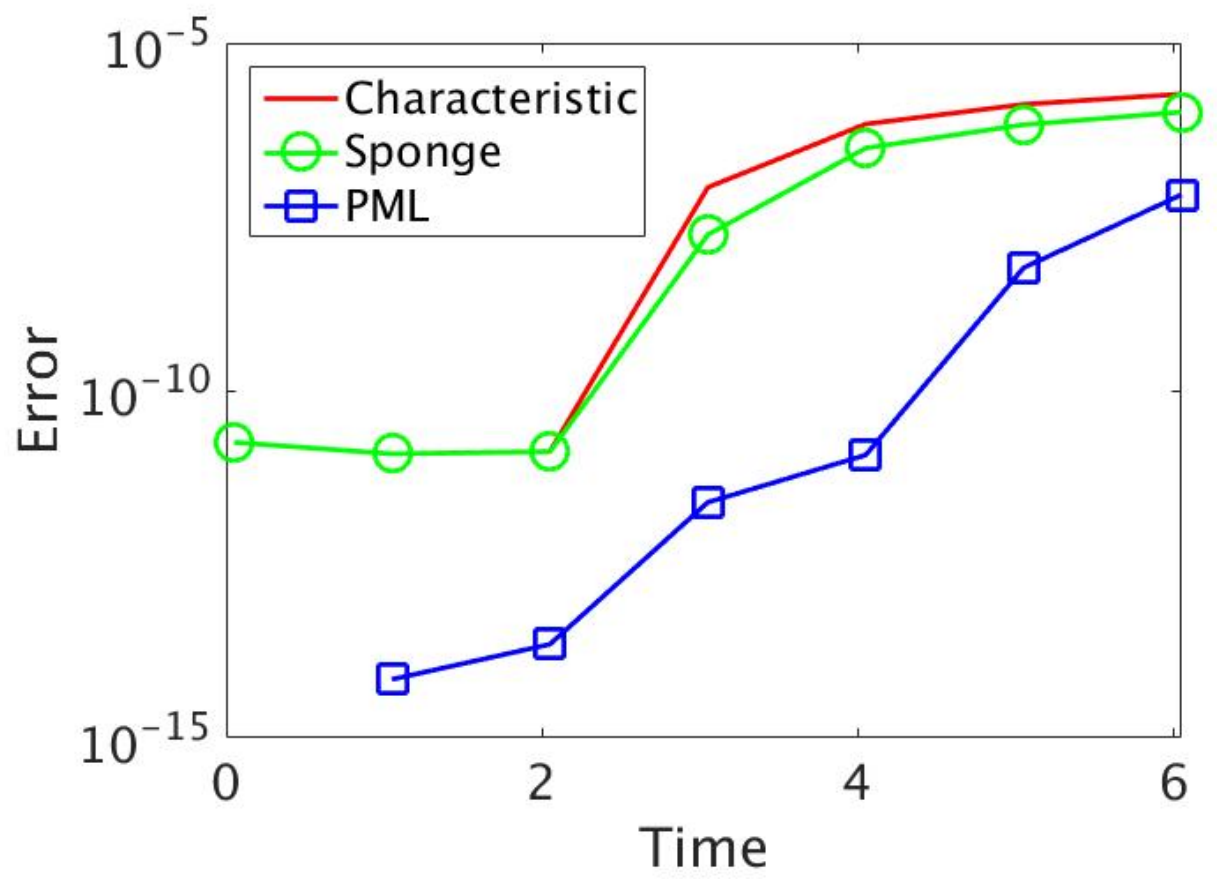

Figure 2. Temporal evolution of the error in pressure distribution between the finite and infinite domain simulations of an acoustic pulse.

The temporal evolution of the root-mean-square error in the pressure distribution between the finite and infinite domain simulations using the three different boundary condition approaches shows that the PML technique performs the best in terms of minimizing any spurious reflections (Figure 2). The simulation with the characteristic boundary condition shows strong reflections (Figure 3-a) from the artificial boundary depending on the incidence angle of the disturbance. The strength of the spurious reflections is low when the incident disturbance is almost normal to the boundary, and increases with the incidence angle. Reflections from the interface can also be seen with the sponge layer (Figure 3-b). For the PML simulations (Figure 3 -c), almost no spurious reflections can be observed.

\section{B. Isentropic vortex convection}

For the second test case, we consider an isentropic vortex in an inviscid fluid convecting in the domain $-3 \leq x_{1} \leq 3 ;-7.5 \leq x_{2} \leq 7.5$, using 12 and 30 elements in the $x_{1}$ and $x_{2}$ directions, respectively. The 


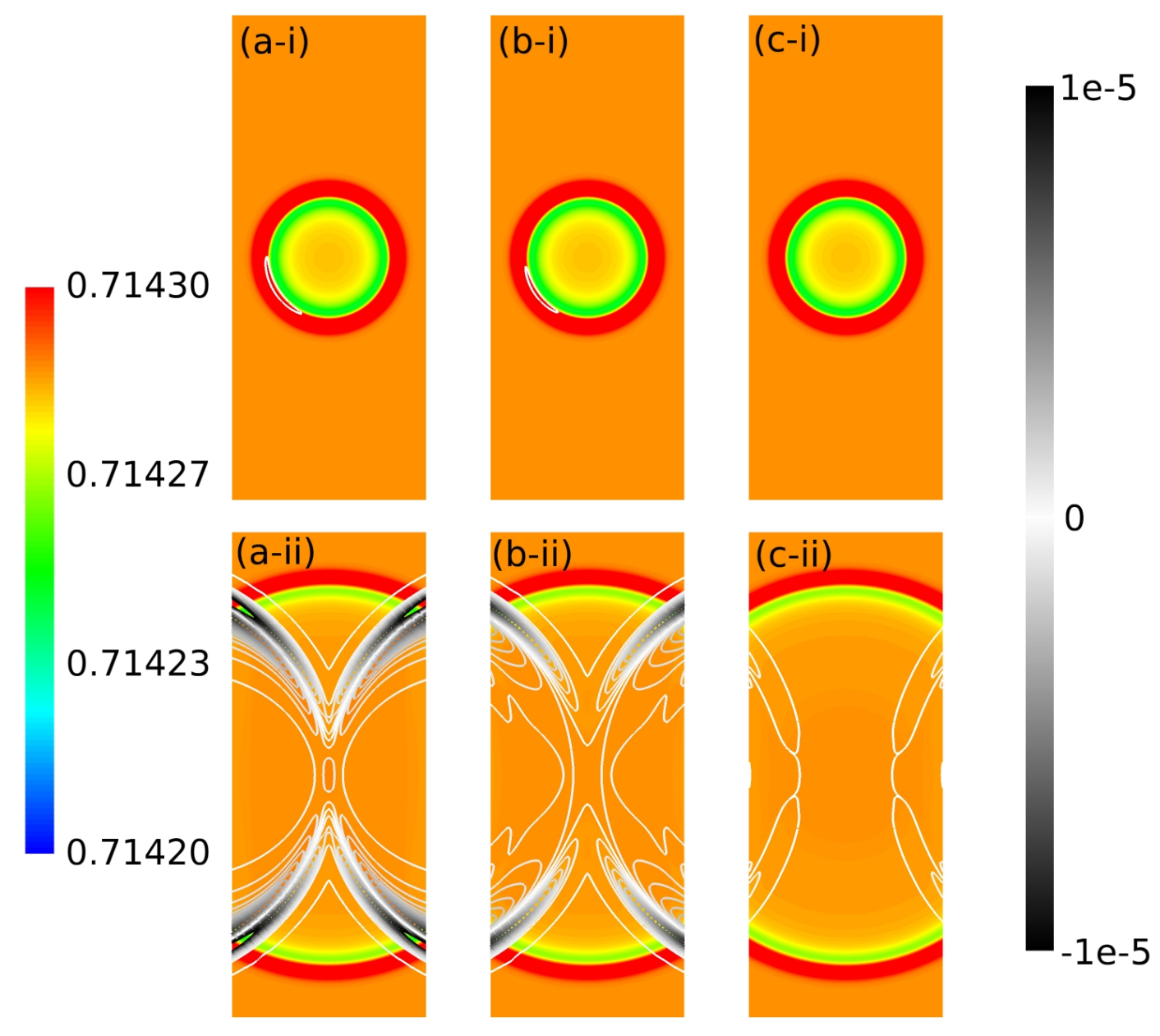

Figure 3. Simulated pressure distribution (in color) and the difference in pressure distributions between the finite and infinite domain simulations (black-white contours) of an acoustic pulse at time $=6$ with (a) characteristic, (b) sponge, and (c) PML boundary conditions for (i) time $=2$, and (ii) time $=6$. Only the domain of interest without the buffer regions is shown in $(b-c)$.

initial condition was prescribed as:

$$
\begin{gathered}
\delta u_{1}=-M_{\infty} \Gamma \frac{x_{2}}{R} \exp ^{-\frac{x_{1}^{2}+x_{2}^{2}}{2}}, \\
\delta u_{2}=-M_{\infty} \Gamma \frac{x_{1}}{R} \exp ^{-\frac{x_{1}^{2}+x_{2}^{2}}{2}}, \\
\delta\left(\frac{p}{\rho}\right)=\frac{1}{2} \frac{\gamma}{\gamma-1} M_{\infty}^{2} \Gamma^{2} \exp ^{-\left(x_{1}^{2}+x_{2}^{2}\right)},
\end{gathered}
$$

where $M_{\infty}$ is the convecting speed of the vortex, $\Gamma=\frac{1}{5}$ is the vortex strength, and $R=0.05$ is the characteristic radius. Two different reference states were considered to test the linear stability of the PML technique for flows that were either unidirectional or oblique in the mean. The first case, with flow only in $x_{1}$ direction, i.e., $\rho_{\infty}, u_{1 \infty}=M_{\infty}=0.1, u_{2 \infty}=0$, and $p_{\infty}$, will be referred to as Case I; the second, with flow in both $x_{1}$ and $x_{2}$ directions, i.e., $\rho_{\infty}, u_{1 \infty}=u_{2 \infty}=0.5$, and $p_{\infty}$, will be referred to as Case II. In Case I, two buffer domains of unit width were added to the left of $x_{1}=-3$ and to the right of $x_{1}=3$ using 2 elements in $x_{1}$ direction. In case II, buffer domains of width $=1$ ( 2 elements in the $x_{1}$ direction) and buffer width $=2$ ( 4 elements in the $x_{1}$ direction) were added to the left of $x_{1}=-3$ and to the right of $x_{1}=3$, respectively. Periodic boundary conditions were used in both the $x_{2}$ and $x_{3}$ directions. The target states at the buffer region were constructed using $\rho_{\infty}, \boldsymbol{u}_{j \infty}$, and $p_{\infty}$. The absorption coefficient was chosen as $\sigma=1$ 
for both the sponge layer and the PML simulations. The PML parameter $\beta$ was calculated as described in Section II. For Case I we set $V_{0}=0$ and $W_{0}=0$, and for Case II we set $V_{0}=0.5$ and $W_{0}=0$. An artificial boundary condition can be considered ideal if the vortex maintained its shape without any distortion as it approached and passed through the boundary $x_{1}=3$.

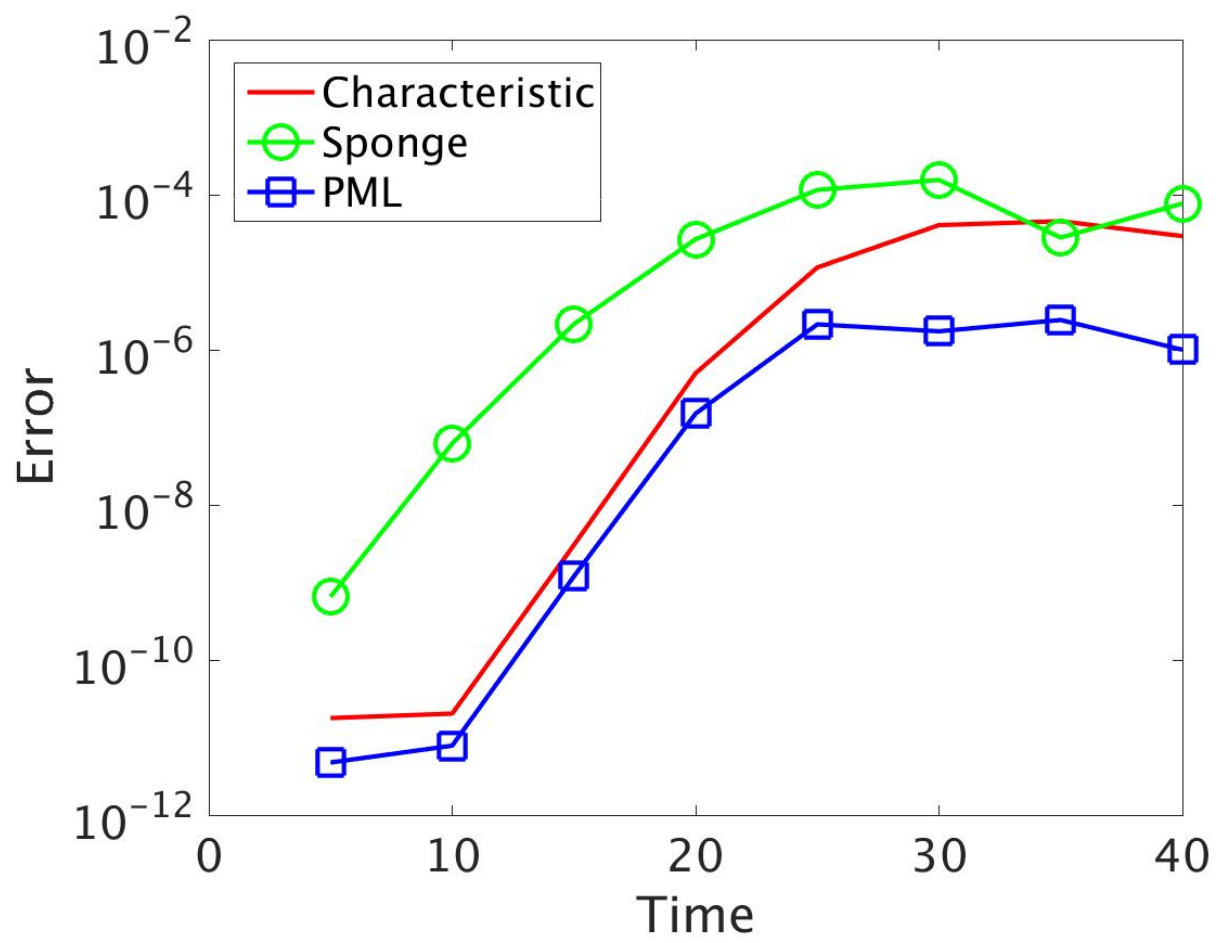

Figure 4. Temporal evolution of the error in pressure distribution between the finite and infinite domain simulations for a vortex convecting in the $x_{1}$ direction.

For Case I, the PML simulation results in the smallest root-mean-square error in the pressure distribution relative to the infinite domain simulation (Figures 4). The simulation using characteristic boundary conditions shows strong variations in the shape of the vortex as it passes through the plane of the boundary (Figures 5-a) and low and high pressure regions can be seen developing as the vortex propagates through the boundary. For the sponge layer simulation (Figures 5-b), the shape of vortex is even more distorted than when the characteristic boundary condition is used. The results with the PML simulation are clearly superior, and show almost no spurious low or high pressure regions and no variation in the shape of the momentum contours (Figures $5-\mathrm{c}$ ). However, similar to the observations by $\mathrm{Hu},{ }^{14,17}$ numerical instabilities were observed when the PML simulations were performed using very large values of $\sigma$.

For Case II, the PML simulations were stable only when $V_{0}$ was set to the bulk velocity in the $x_{2}$ direction, as observed by Parrish \& Hu. ${ }^{20}$ The results with the PML are superior to those obtained with the other boundary conditions for this case as well (Figure 6). The root-mean-square error in pressure is higher overall for Case II in comparison to Case I since the vortex strength is higher. Within the PML region all acoustic perturbations are damped exponentially with the exponent taking the form $-\frac{\sigma(M+\cos \phi)}{\left(1-M^{2}\right)(1+M \cos \phi)} x_{1}$; and the vorticity and entropy waves are damped exponentially as $-\frac{\sigma}{\left(1-M^{2}\right) M} x_{1}$, where $M$ is the characteristic velocity in the $x_{1}$ direction and $\phi$ is the angle between the wave-front normal vector and the $x_{1}$ direction. ${ }^{14}$ In order to account for this, we have increased the length of the buffer region to twice for the Case II simulation. Strong acoustic perturbations are noticed for the characteristic boundary condition and sponge simulations in comparison to the PML simulations (Figure 7). These results are similar to those obtained by Parrish \& $\mathrm{Hu}^{20}$ 

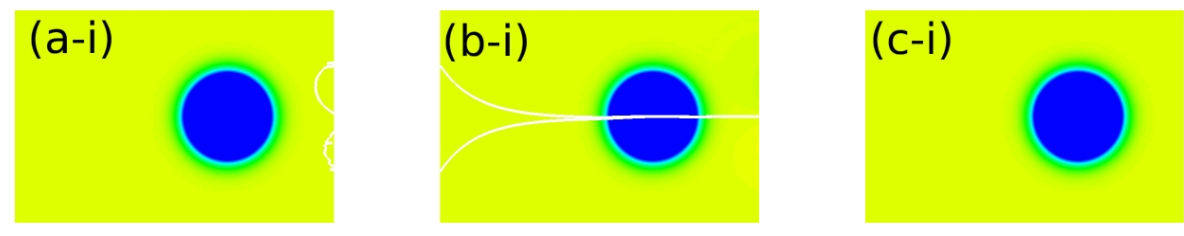

0.71430
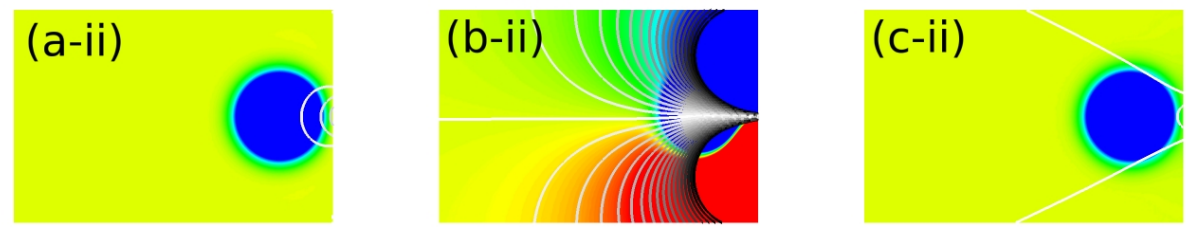

0.71429
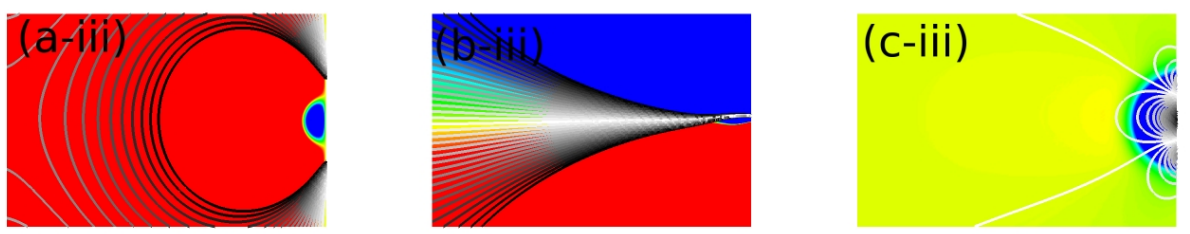

0.71426
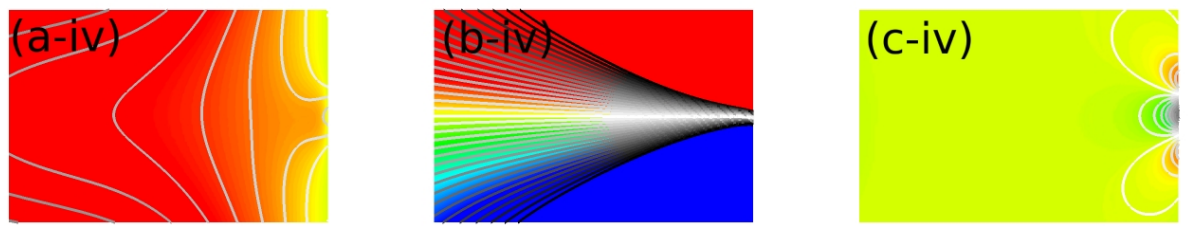

0.71428

0.71425

\section{$-5 e-5$}

Figure 5. Temporal evolution of the pressure distribution (in color) and the difference in the pressure distributions between the finite and infinite domain simulations (in black-white contours) for a vortex convecting in the $x_{1}$ direction with (a) characteristic, (b) sponge, and (c) PML boundary conditions. In (i) the vortex is far from the $x_{1}=3$ plane, time $=10$ (ii) the vortex is about to cross the $x_{1}=3$ plane, time $=20$; (iii) half of the vortex has crossed the $x_{1}=3$ plane, time $=30$; and (iv) the entire vortex has crossed the $x_{1}=3$ plane, time $=40$. Only the domain of interest without the buffer regions is shown in $(\mathbf{b}-\mathbf{c})$. 


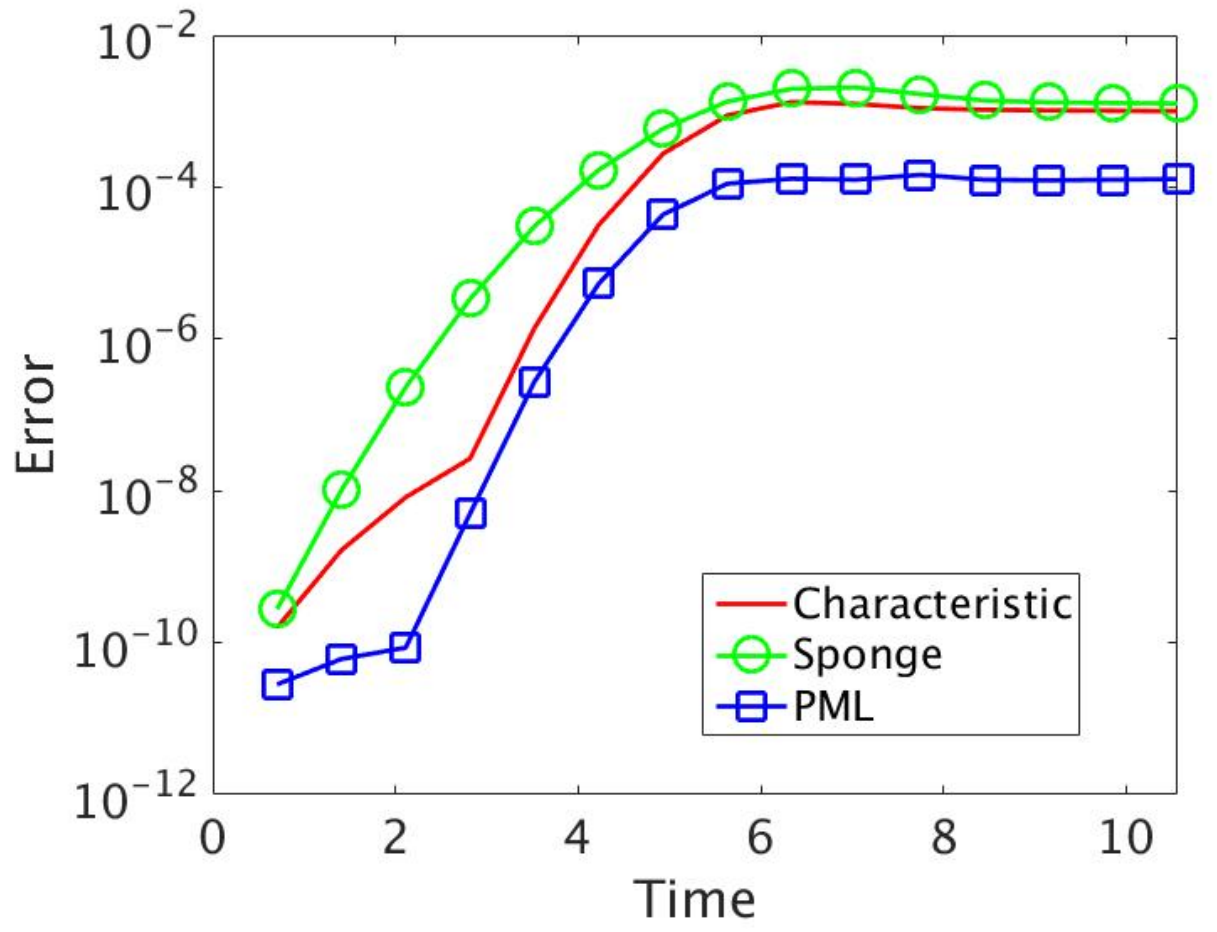

Figure 6. Temporal evolution of the error in the pressure distribution between the finite and infinite domain simulations for a vortex convecting in both the $x_{1}$ and $x_{2}$ directions. 

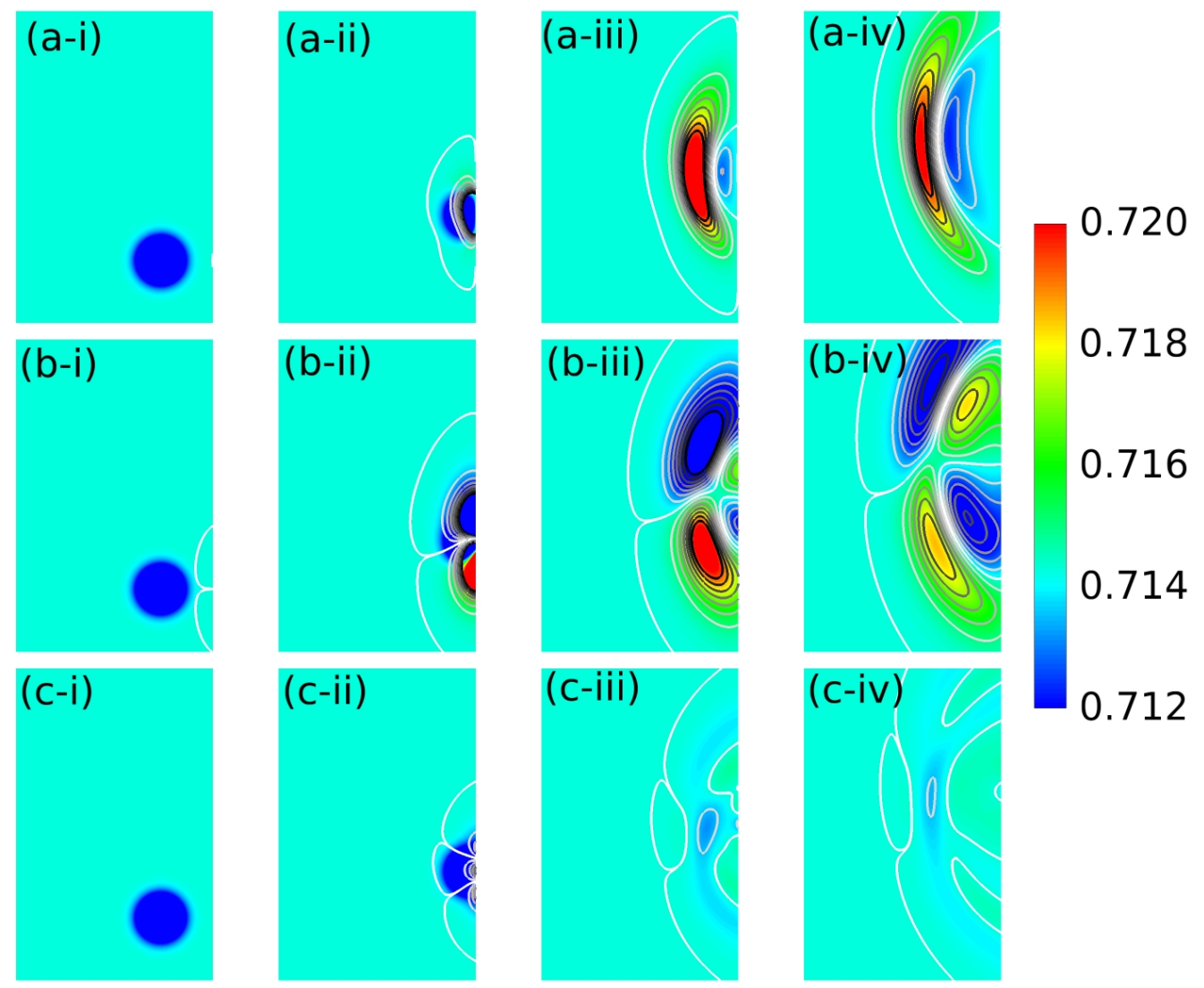

0.720

$-5 \mathrm{e}-3$

0

$5 e-3$

Figure 7. Temporal evolution of the pressure distribution (in color) and the difference in the pressure distributions between the finite and infinite domain simulations (in black-white contours) for a vortex convecting in both the $x_{1}$ and $x_{2}$ directions with (a) characteristic, (b) sponge, and (c) PML boundary conditions. In (i) the vortex is about to cross the $x_{1}=3$ plane, time $=2.8$; (ii) half of the vortex has crossed the $x_{1}=3$ plane, time $=5.6$; (iii) the entire vortex has crossed the $x_{1}=3$ plane, time $=8.4$; and (iv) after the entire vortex has crossed the $x_{1}=3$ plane, time $=10.56$. Only the domain of interest without the buffer regions is shown in $(\mathrm{b}-\mathrm{c})$. 

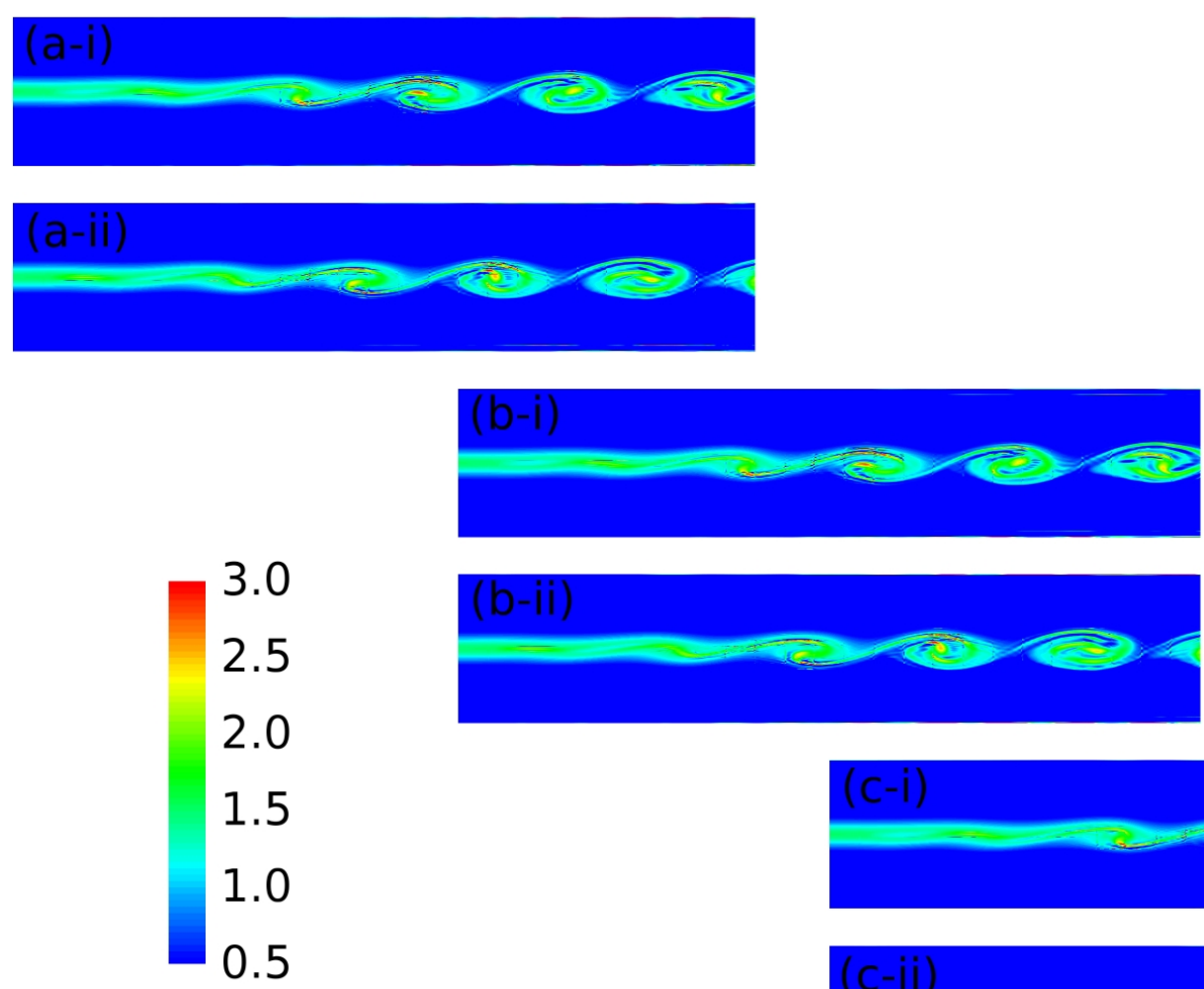

1.5
1.0
0.5
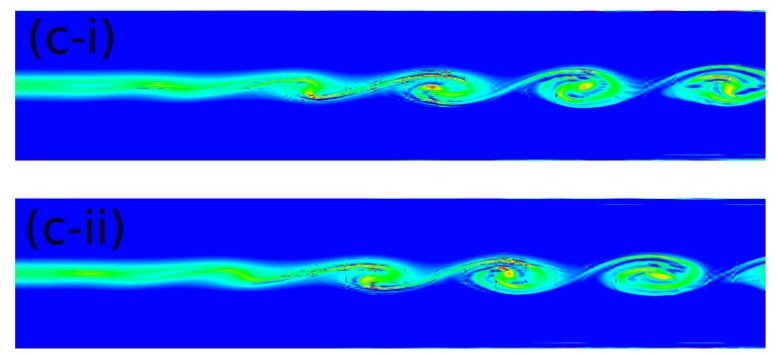

Figure 8. Contours of the vorticity magnitude at different time instants for the shear layer simulations with (a) characteristic, (b) sponge, and (c) PML boundary conditions. Only the domain of interest without the buffer regions is shown in $(b-c)$.

\section{Shear layer}

We next consider the phenomenon of inviscid vortex roll-up in a 2-D shear layer flow induced by the KelvinHelmholtz instability in the computational domain $-1 \leq x_{1} \leq 9 ;-1 \leq x_{2} \leq 1$ with 25 elements each in the $x_{1}$ and $x_{2}$ directions. The prescribed initial conditions are given as:

$$
\left[\begin{array}{c}
\rho \\
u_{1} \\
u_{2} \\
p
\end{array}\right]=\left[\begin{array}{c}
\bar{\rho}\left(x_{2}\right) \\
\bar{U}\left(x_{2}\right) \\
0 \\
\underline{1}
\end{array}\right]
$$

where

$$
\begin{gathered}
\bar{U}\left(x_{2}\right)=\frac{1}{2}\left[\left(U_{1}+U_{2}\right)+\left(U_{1}-U_{2}\right) \tanh \left(\frac{2 x_{2}}{\delta}\right)\right], \\
\rho\left(x_{2}\right)=\frac{1}{T_{1} \frac{\bar{U}-U_{2}}{U_{1}-U_{2}}+T_{2} \frac{U_{1}-\bar{U}}{U_{1}-U_{2}}+\frac{\gamma-1}{2}\left(U_{1}-\bar{U}\right)\left(\bar{U}-U_{2}\right)},
\end{gathered}
$$


with $U_{1}=0.8, U_{2}=0.2, \delta=0.4, T_{1}=1, T_{2}=0.8$. In order to excite the Kelvin-Helmholtz instability, a source function of the form $5 \sin \left(\frac{\pi}{2} t\right) \exp ^{-\log (2) \frac{\left(x_{1}+0.5\right)^{2}+\left(x_{2}-0\right)^{2}}{0.03^{2}}}$ was included in the energy equation. Characteristic and periodic boundary conditions were used in $x_{2}$ and $x_{3}$ directions, respectively. For simulations with both the sponge layer and the PML, buffer regions of width 0.5 ( 1 element in $x_{1}$ direction) were added at $x_{1} \leq-1$ and $x_{1} \geq 9$. A value of $\sigma=10$ was used for both simulations.
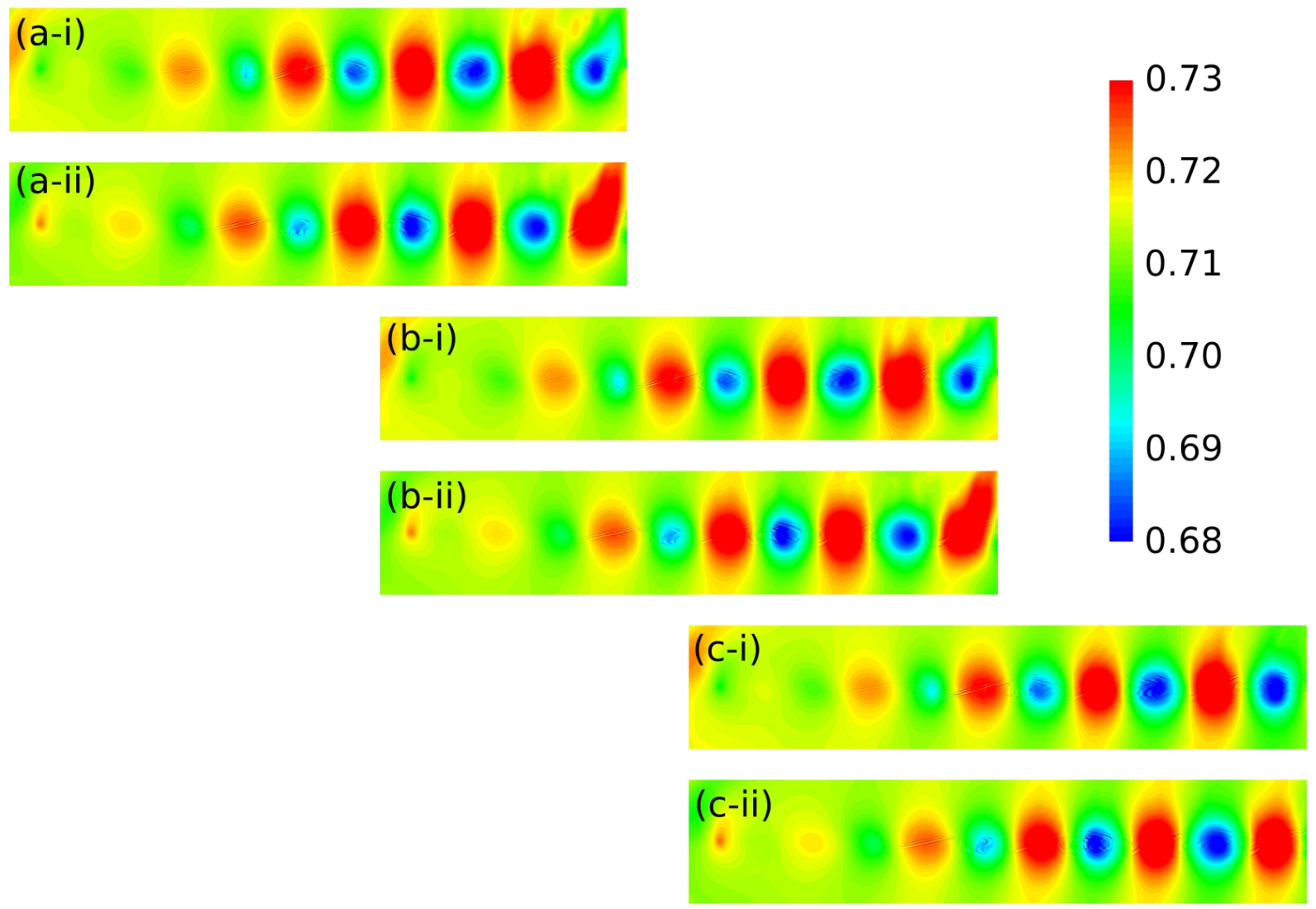

Figure 9. Contours of the pressure at different time instants for the shear layer simulations with (a) characteristic, (b) sponge, and (c) PML boundary conditions. Only the domain of interest without the buffer regions is shown in $(b-c)$.

For these simulations, the target states in the buffer regions were constructed using the initial conditions. Following $\mathrm{Hu},{ }^{17}$ a value of $\beta=1 / 1.4$ was used, which is close to the value of $\beta$ calculated using the $x_{1}$ bulk velocity. The quantities $V_{0}=0, W_{0}=0$ were prescribed since there were no cross flow-components for the target state in the buffer regions.

The choice of characteristic, sponge, or PML boundary conditions seems to have minimal effect on the variation of the vorticity magnitude as the vortex passes through the $x_{1}=9$ plane (Figure 8 ). However, the effect of the boundary condition is more noticeable in plots of the pressure for the same periods (Figure 9). With both the characteristic and the sponge boundary condition strong spurious reflections are seen. These reflections are barely noticeable for the PML case. These spurious reflections are also observed in the value of the mean (time-averaged) pressure. Figure 10 shows the time-averaged pressure for the characteristic and PML boundary conditions obtained by averaging over two domain flow-through times. Reflections contaminate the flow and produce low pressure regions near the outflow boundary with the characteristic boundary condition. With the PML technique, the mean pressure varies smoothly except in a very localized 
region close to the $x_{1}=9$ plane. Note that, the effect of these localized mean pressure variation is not noticed over the bulk of the domain of interest.
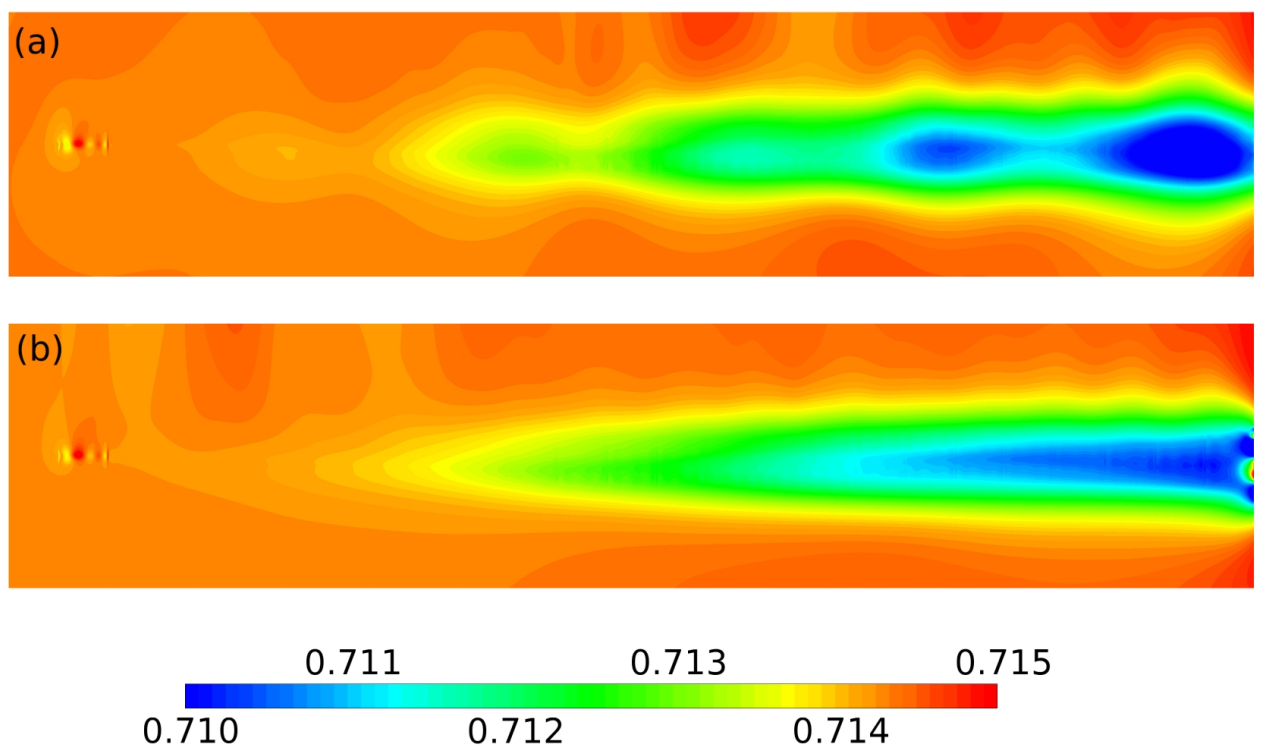

Figure 10. Time-averaged pressure (averaged over two domain flow-through times) for the shear layer simulations with (a) characteristic, and (b) PML boundary conditions. Only the domain of interest without the buffer regions is shown in (b).

As mentioned in Section I, the complete state of the flow at the outflow boundary in most practical flows is often not known. For these cases, a target state at the outflow buffer region needs to be constructed. We have tested different approaches for this purpose. One approach is to set the target state from the initial condition, but this may not be entirely accurate for the present test case where the shear layer thickens due to vortex roll-up. Another approach is to conduct a precursor simulation with the outflow buffer region excluded to gather mean flow statistics, and then use this computed mean flow as a target state for subsequent simulations with the buffer region included. Although the constructed target state may be corrupted by spurious numerical reflections, this approach still provides reasonable mean flow criteria (equation (2)), and is likely to be a better choice than any other crude guess that is based on, say, conserving the mass flow rate. No difference in the numerical results for this test case were noticed using these two different target flows for the PML simulations.

\section{Low-pressure turbine cascade}

In addition to the above canonical flow simulations, we have applied the PML technique to simulate flows in realistic configurations, viz., subsonic flows in low-pressure turbine cascades. We first consider the welldocumented lightly loaded T106A cascade with $R e=60000$, based on isentropic exit speed and chord length, and in the absence of any inflow turbulence. Details of the geometry and flow configuration can be found in Garai et al. ${ }^{25}$ The $h-p$ convergence study was performed by Garai et al., ${ }^{25}$ and the results using the finest elements are presented here. Buffer regions extending about one quarter axial-chord length (with two elements in $x_{1}$ direction) were added at the inflow and outflow boundary. The absorption coefficient, $\sigma$, was chosen as 10. At the inflow buffer region, the target state was set to the inflow condition. As the full state at the outflow was not known from the experimental data, a precursor simulation spanning 4 domain 
flow-through times was first performed, and mean flow statistics were gathered for the last 2 domain flowthrough times. This calculated mean flow was then used as the target state for the outflow buffer region for the simulations using both the sponge layer and PML technique. Periodic boundary conditions were used in the pitch and span directions. The PML parameters, $\beta, V_{0}$, and $W_{0}$, were calculated using inflow conditions for the inflow PML, and using the calculated mean from the precursor simulations for the outflow PML.

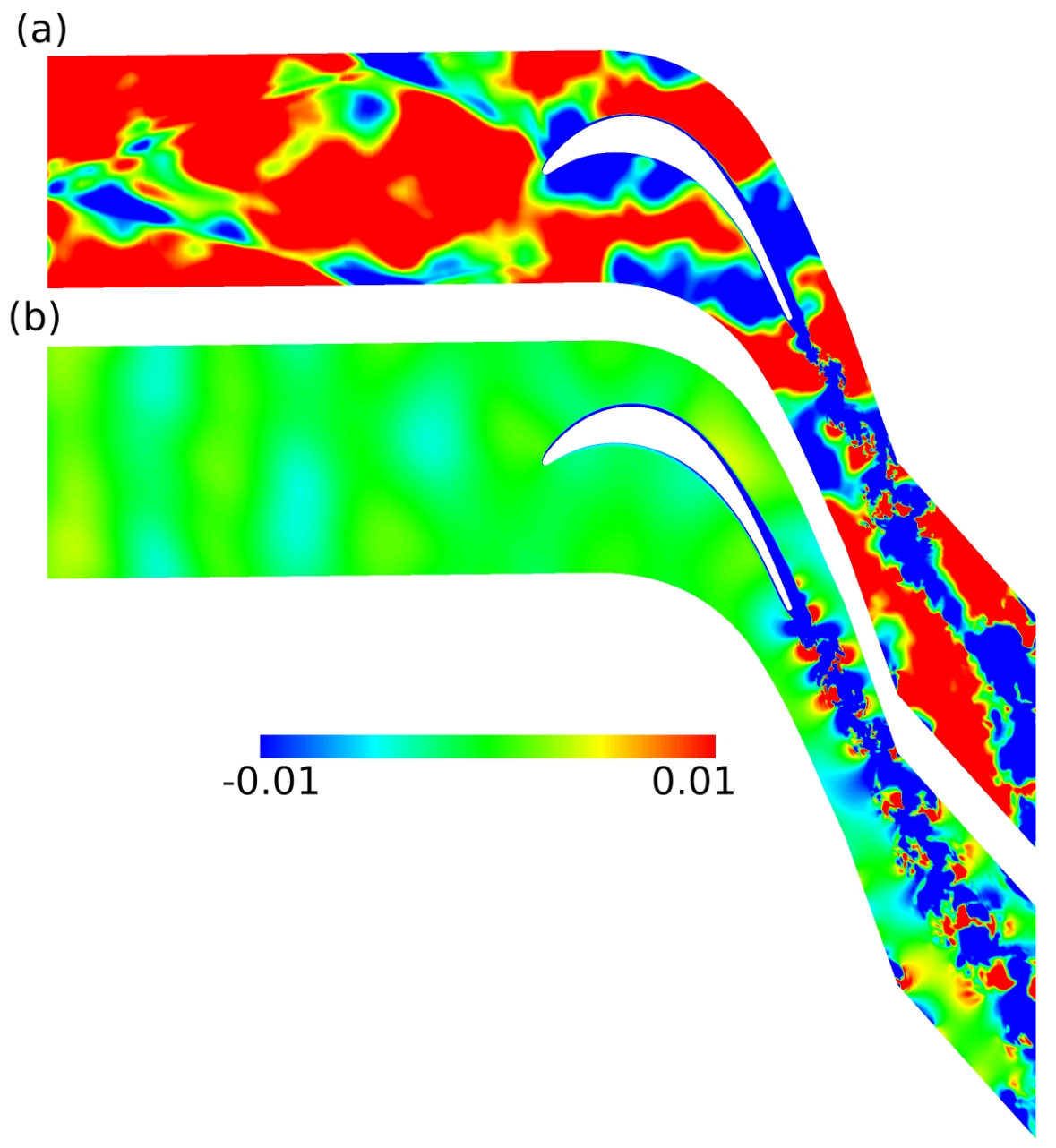

Figure 11. Contours of the total pressure difference $\left(\frac{p_{t, i n f l o w}-p_{t}}{p_{t, i n f l o w}}\right)$ for the T106A low-pressure turbine cascade simulation with (a) characteristic, and (b) PML boundary conditions. Only the domain of interest without the buffer regions is shown in (b).

The variation of the total pressure with respect to the inflow total pressure is plotted for both the characteristic and the PML boundary condition in Figure 11. Spurious reflections from non-normal disturbances incident on the inflow plane are clearly evident in the simulation with characteristic boundary conditions (Figure 11-a). These spurious reflections are very slow to die down since they reflect back and forth between the inflow boundary and the airfoil. These reflections tend to increase the total pressure with respect to the inflow condition, and affect the vortex shedding process at the stationary state. On the other hand, the PML boundary condition damps out the numerical acoustic disturbances with no spurious reflections (Figure 11-b), and the total pressure equilibrates with the inflow condition when the stationary state is realized. Application of the PML technique changes the span and temporal averaged surface pressure distribution marginally, in a very small region close to the trailing edge (Figure 12).

The second low-pressure turbine cascade considered is the T106C configuration where the airfoil geometry is the same as the T106A case but the airfoil pitch is increased to achieve higher loadings. In this configuration, a large separation bubble develops on the aft portion of the suction side that must be ac- 


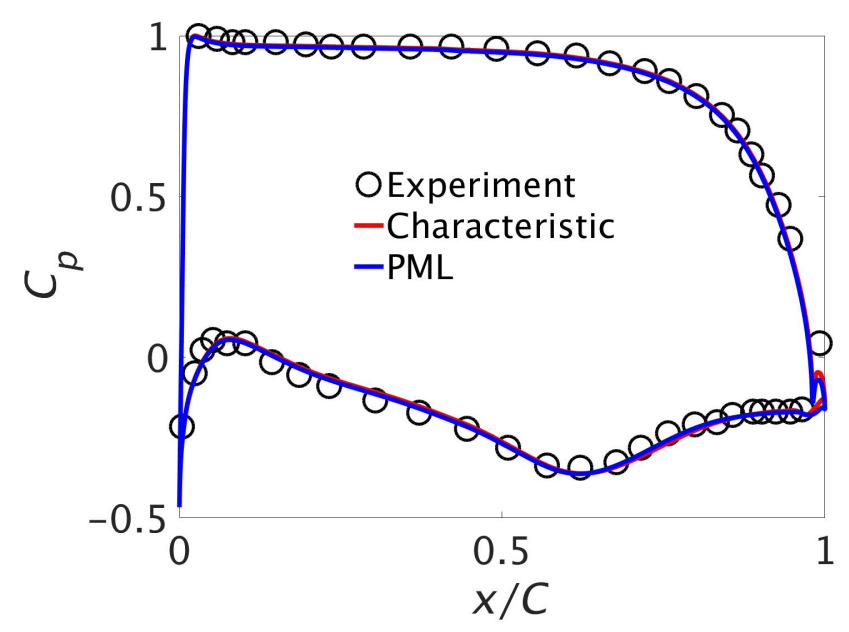

Figure 12. $C p$ distribution for the T106A airfoil configuration.

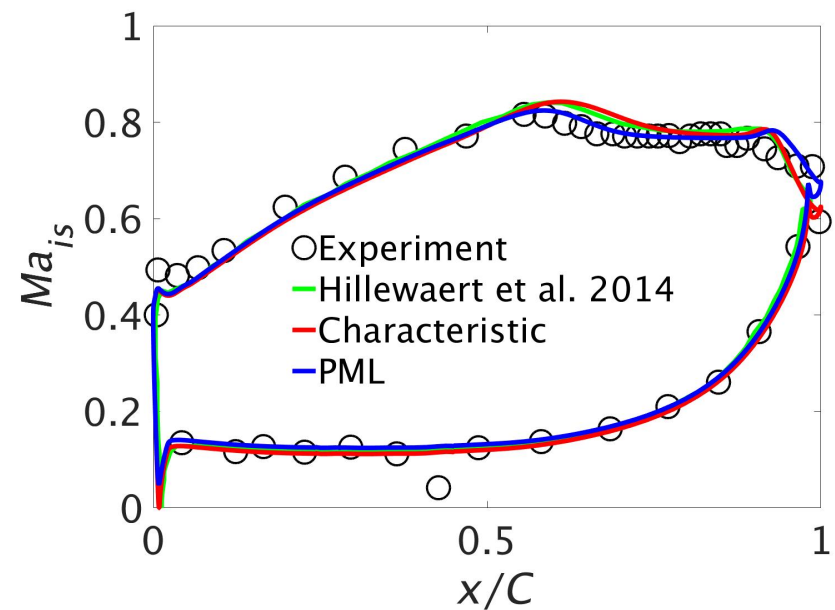

Figure 13. Isentropic Mach number distribution for the T106C airfoil configuration.

curately captured. We consider the $R e=80000$ case for which both experimental and other DNS results are available in the literature. Note that the operating condition for the $\mathrm{T} 106 \mathrm{C}$ case is higher than for the T106A case. Elements similar to the T106A case were used for the T106C case, and marginal changes in the surface pressures averaged in both span and time were noticed when spatialy 4 th or 8 th-order elements were used. As a first attempt, we have used the same buffer strength and length as before. Numerical instabilities (non-zero $\boldsymbol{Q}$ ) were noticed at the outflow PML boundaries when the simulations were run for long time intervals (about 5 domain flow-through time). However, these instabilities did not occur when the buffer strength was increased to $\sigma=50$ for 6 domain flow-through times.

The simulation results using the characteristic boundary condition for the T106C configuration are comparable to those documented in the $2^{\text {nd }}$ workshop on high order methods,${ }^{26}$ and in Hillewaert et al. ${ }^{27}$ When the PML technique is used the isentropic Mach number $\left(M a_{i s}=\sqrt{\frac{2}{\gamma-1}\left[\left(\frac{p_{t 1}}{p}\right)^{\frac{\gamma-1}{\gamma}}-1\right]}\right)$ distribution is closer to the experimental data (Figure 13). The present simulations overestimate the surface pressures in the fore region of the suction side. However, these results are consistent with those obtained by Hillewaert et al. ${ }^{27}$ who conjectured that this mismatch in the pressures may be due to the incorrect flow angle and blade stagger angle reported in the experiments. Downstream of the suction peak in the laminar separation 


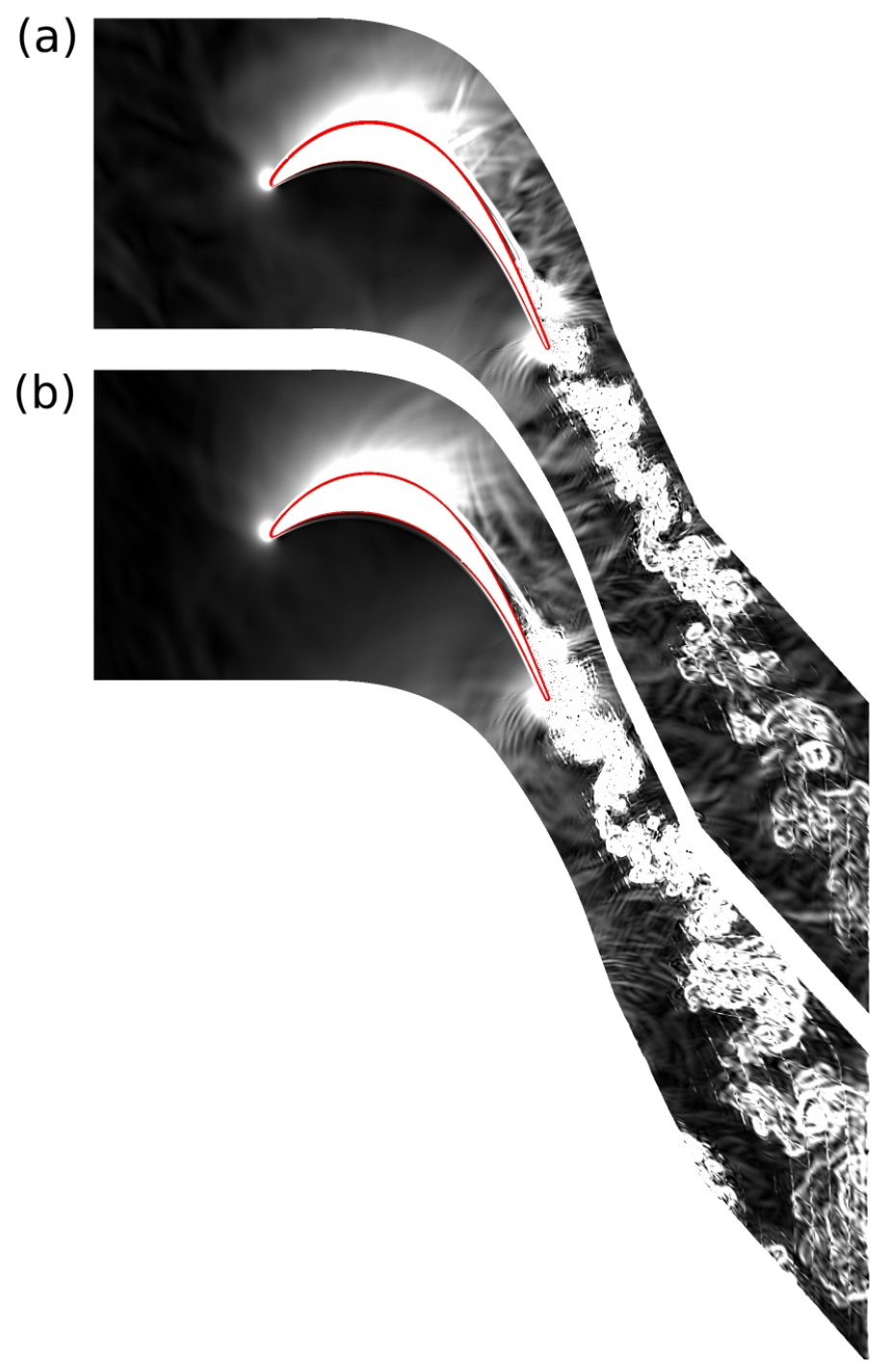

Figure 14. Contours of density gradient $\left(\sqrt{\frac{\partial \rho}{\partial x}}^{2}+\frac{\partial \rho}{\partial y}^{2}\right)$ for the T106C low-pressure turbine cascade simulation with (a) characteristic, and (b) PML boundary conditions. Only the domain of interest without the buffer regions is shown in $(b)$.

bubble region, the results from the present simulation are in better agreement with the experiment than the results of Hillewaert et al. ${ }^{27}$ For the simulations using the characteristic boundary condition, spurious reflections overwhelm the physical acoustic waves, and the size of the suction-side laminar separation bubble is diminished. With the PML boundary condition there are no spurious reflections and only the physical acoustic waves emanating from vortex shedding at the trailing edge can be noticed in Figure 14.

\section{E. DNS of flow around a solid block}

We used the current PML technique to perform DNS of flow around a solid block in support of wind tunnel experiments being performed in the Fluid Mechanics Laboratory at NASA Ames Research Center. The Mach number and the Reynolds number in these simulations are 0.45 and 5000, respectively. The wind tunnel experiment is described in detail in Roozeboom et al. ${ }^{28}$ Figure 15 plots the instantaneous iso-contours of total pressure around the solid block and in its wake along the centerline of the wind tunnel obtained using both the characteristic and PML boundary conditions. The PML technique reduces the spurious reflections from the outflow boundary that can be otherwise be clearly seen in the simulation with the characteristic 
boundary condition.

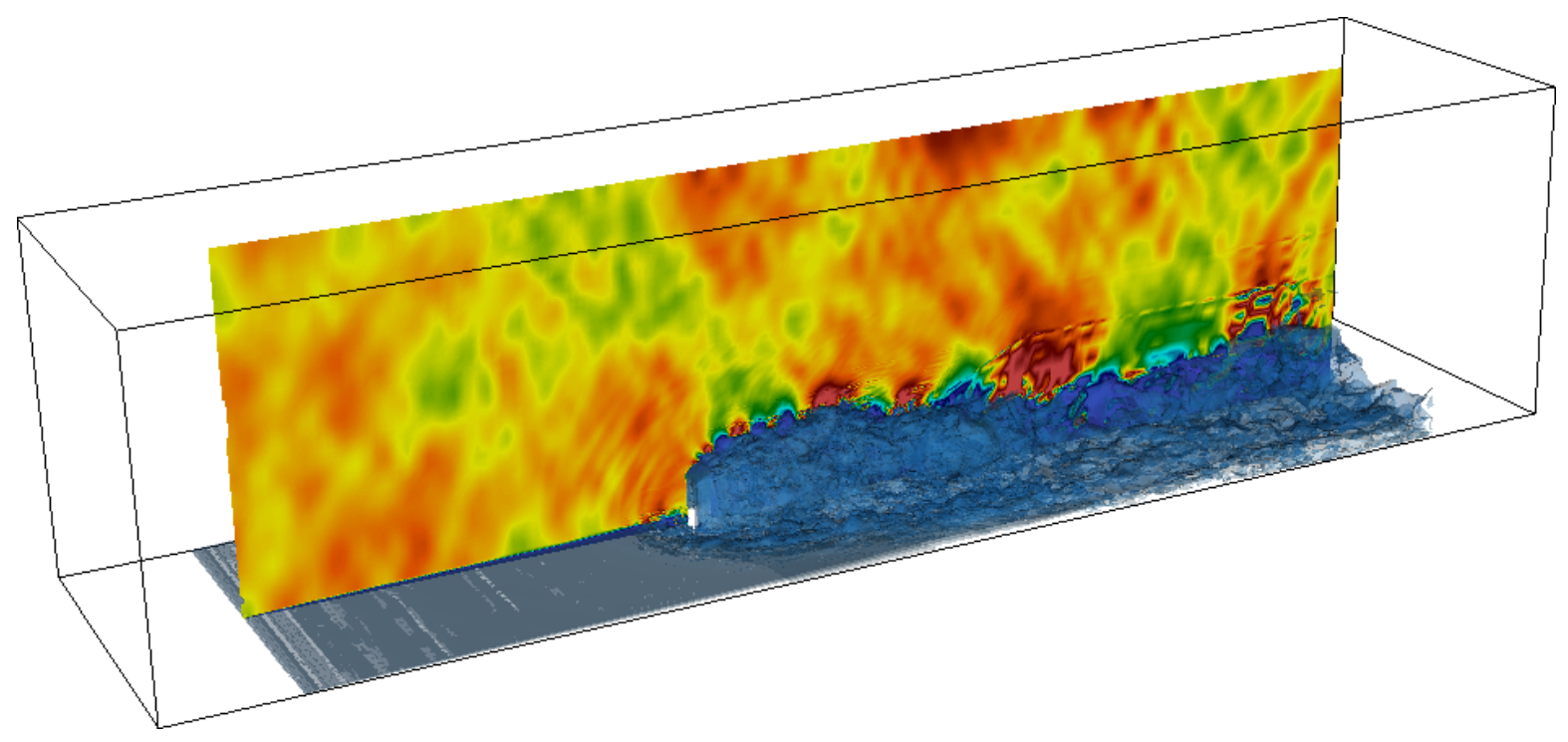

(a) Characteristic boundary condition

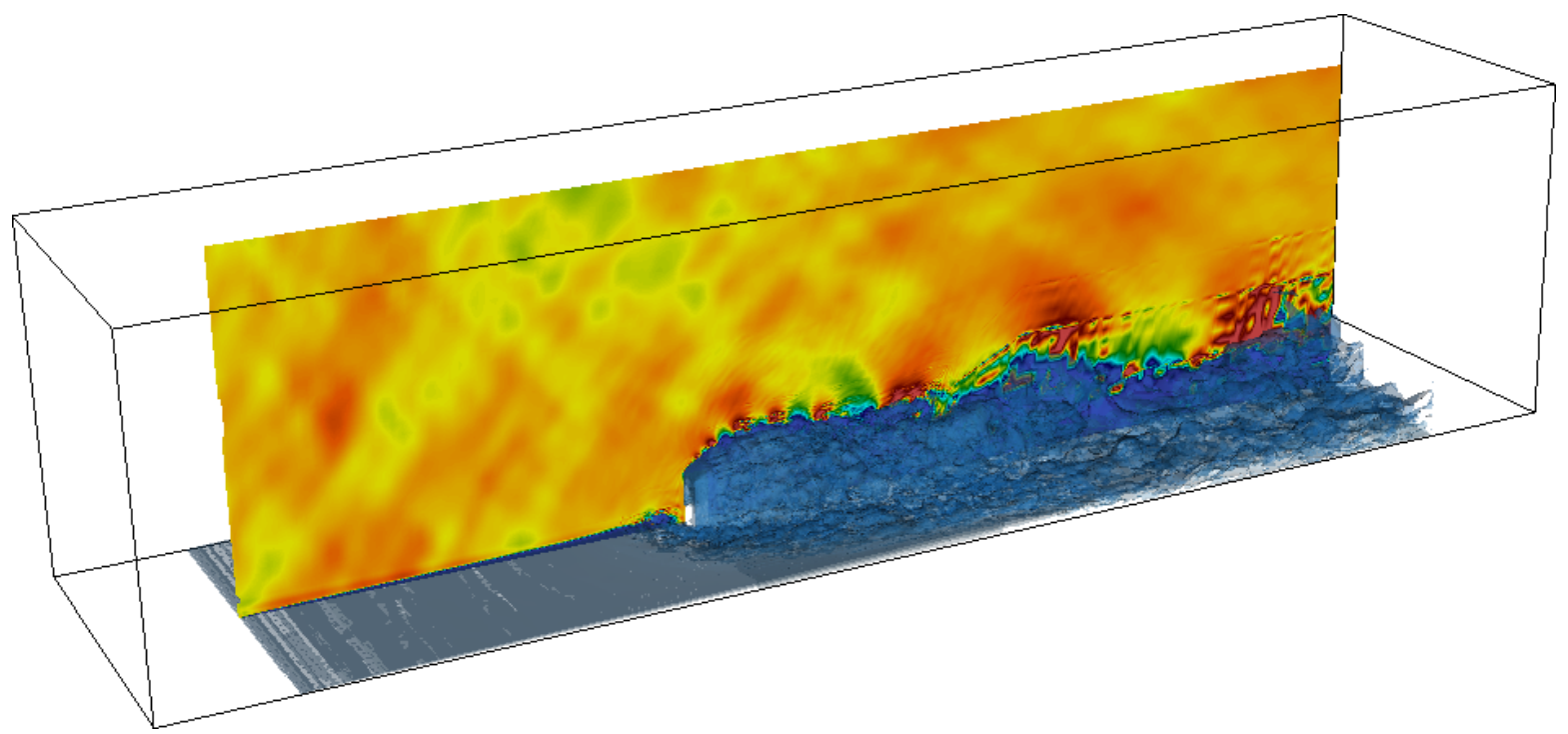

(b) PML

Figure 15. Total pressure and iso-contours of Mach number for flow over a solid block. Only the domain of interest without the buffer regions is shown in (b).

Unfortunately, the simulation using the PML boundary condition became unstable after it had progressed for a long time interval (after two domain flow-through time), as the value of the auxiliary PML variable $(\boldsymbol{Q})$ grew unbounded at the edge of the boundary layer in the outflow PML region. In this region of the flow the instantaneous velocity perturbations increased in magnitude to several times the mean velocity and even reversed flow was observed in the boundary layer. This behavior could not be prevented even though we tried using a wide range of values for $\sigma$ (ranging from 1 to 100).

\section{Stability of the PML equations}

The stability of the PML equations can be derived from linear stability theory with uniform flow, ${ }^{14}$ as mentioned in Sections II, III. We believe that the failure of the PML simulations over long time intervals for the T106C cascade (using a lower value of $\sigma$ ) and the flow around a solid block is due to strong nonlinear 
effects. In this section we examine the nonlinear behavior of the PML equations in the presence of large perturbations by revisiting the convecting isentropic vortex test case but with large perturbations. The results shown are at a freestream Mach number of 0.1 and using three different vortex strengths corresponding to velocity perturbations that are approximately $10 \%, 100 \%$, and $500 \%$ of the freestream value. Figure 16 shows the evolution of x-momentum as the vortex convects through the PML region. When the vortex strength is weak, the PML technique damps the vortex as it travels through the buffer region and reflections are minimized. The state returns to the target value while the $\boldsymbol{Q}$-variable returns to zero once the vortex has passed through the domain. When the vortex strength increases to $100 \%$ of the free-stream value the vortex is damped as it travels through the PML but a large perturbation in the state remains undamped in the PML region for very long times. In practical simulations, further flow perturbations would follow which could amplify the undamped region leading to instability. However, it is interesting to note that in the non-PML region of the domain and, in particular, at the exact PML interface, the state returns to the freestream value. When the vortex strength is increased further, it appears that this remaining perturbation from the transit of the vortex leads to an instability and eventually causes the simulation to blow up.

To further understand the issue, we track the evolution of the conservative state, $\boldsymbol{u}$, auxillary state $\boldsymbol{Q}$, and the terms involved in the evolution of $\boldsymbol{u}$ and $\boldsymbol{Q}$ in the PML region. Figure 17 presents the evolution of the terms corresponding to the x-momentum at quadrature point in the middle of the first element just inside the PML region. For the small vortex we can see how $\boldsymbol{Q}$ tracks $u-\bar{u}$ then both $\boldsymbol{u}$ and $\boldsymbol{Q}$ return to the free-stream value. For the vortex with strength of about $100 \%$ of the free-stream value after the transit of the vortex there is non-zero value of $\boldsymbol{u}$ and $\boldsymbol{Q}$ (corresponding to the solutions plotted in Figure 17). $u_{, t}$ and $Q_{, t}$ are essentially zero since the term $\sigma Q$ is balanced by $F_{x, x}$. For the strongest vortex $\boldsymbol{Q}$ remains strong enough in the PML region to cause an instability and lead to the eventual failure of the simulation.

One potential approach to addressing the nonlinear stability of the PML equations could involve additional forcing to ensure that $\boldsymbol{u}$ and $\boldsymbol{Q}$ return to their nominal values, $\overline{\boldsymbol{u}}$ and 0, respectively. However, such additional forcing would necessarily destroy the non-reflective property of the PML. Another alternative could involve some form of quadratic penalization such that the regular PML behavior is essentially recovered for small perturbations. We have been experimenting with several such formulations but are yet to find a generally applicable solution.

\section{Concluding Remarks}

The PML technique implemented here for a high-order spectral-element DG method shows promise in accurately representing the inflow and outflow boundaries for unsteady simulations in truncated computational domains. Since the PML technique is non-reflective only in the continuous sense, some small reflections are noticed from the interface in the present discrete simulations. The nonreflective and absorptive properties of the PML are independent of the frequency and the angle of the waves. For the test cases considered, the performance of the PML is superior to any other method when the perturbations are small. Although the PML technique requires the solution of additional equations when compared to the characteristic or sponge boundary conditions, the extra computational work is justified by the accurate propagation of acoustic energy in the domain. The results for the turbine cascade are particularly encouraging. The lack of proper non-reflecting inflow and outflow boundary conditions are a major issue in such internal flows where spurious acoustic reflections from the boundaries can often swamp the solution and lead to poor (or non) convergence and inaccurate results. The nonlinear stability of the PML equations remains an open issue for with flows with large perturbations and will need to be addressed before the technique can be broadly applied.

\section{Acknowledgments}

Funding for Anirban Garai was provided by the Advanced Air Transport Technology Project in the NASA Advanced Air Vehicles Program through the NASA Postdoctoral Program administrated by Oak Ridge Associated Universities (ORAU). We also thank NASA Advanced Supercomputing (NAS) facility at NASA Ames Research Center for providing computing resources. 


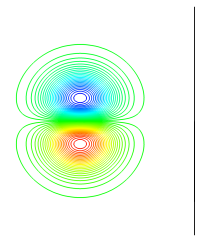

(a) $10 \%$ vortex, $t=0.0$

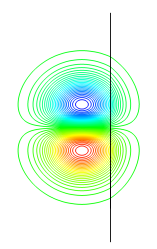

(d) $10 \%$ vortex, $t=3.75$

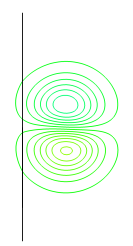

(g) $10 \%$ vortex, $t=7.5$

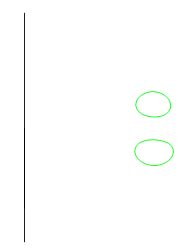

(j) $10 \%$ vortex, $t=11.25$

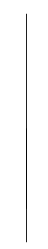

(m) $10 \%$ vortex, $t=15.0$

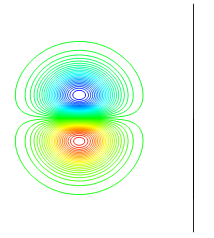

(b) $100 \%$ vortex, $t=0.0$

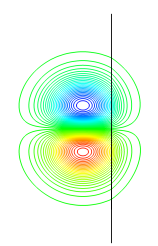

(e) $100 \%$ vortex, $t=3.75$

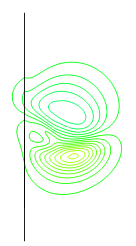

(h) $100 \%$ vortex, $t=7.5$

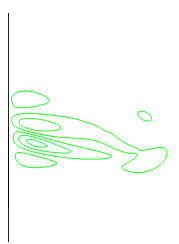

(k) $100 \%$ vortex, $t=11.25$

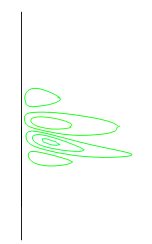

(n) $100 \%$ vortex, $t=15.0$

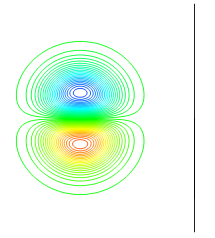

(c) $500 \%$ vortex, $t=0.0$

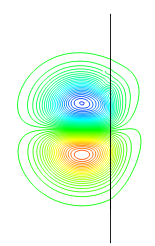

(f) $500 \%$ vortex, $t=3.75$

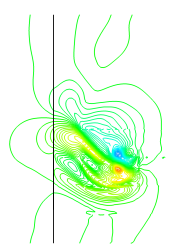

(i) $500 \%$ vortex, $t=7.5$

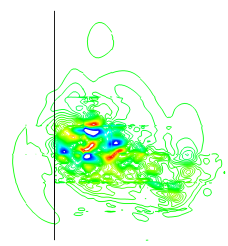

(l) $500 \%$ vortex, $t=11.25$

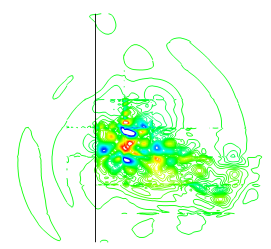

(o) $500 \%$ vortex, $t=15.0$

Figure 16. Isentropic vortex convection through the PML domain. The solid line denotes interface between the fluid and the PML domain. 


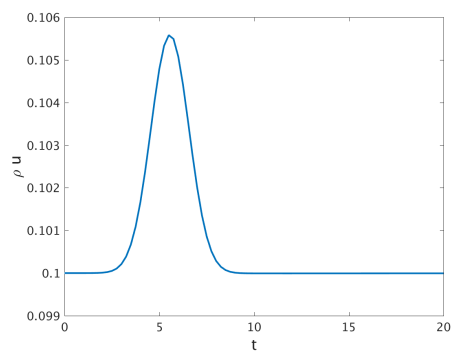

(a) $\boldsymbol{u}, 10 \%$ vortex

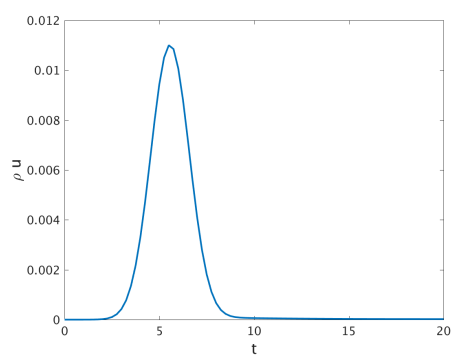

(d) $\boldsymbol{Q}, 10 \%$ vortex

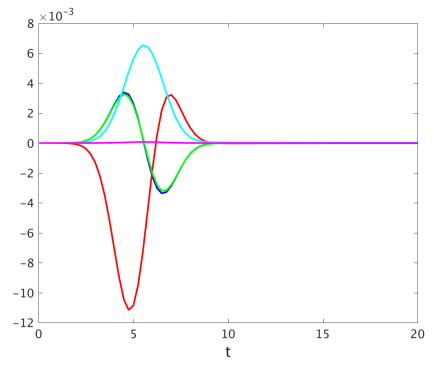

(g) $\boldsymbol{u}$-balance, $10 \%$ vortex

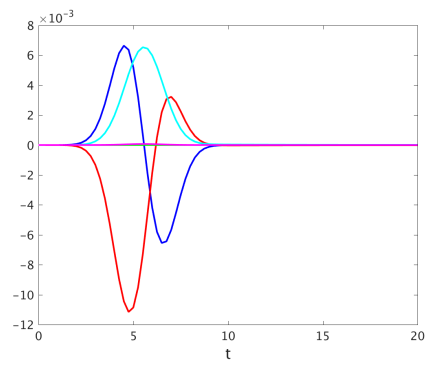

(j) $\boldsymbol{Q}$-balance, $10 \%$ vortex

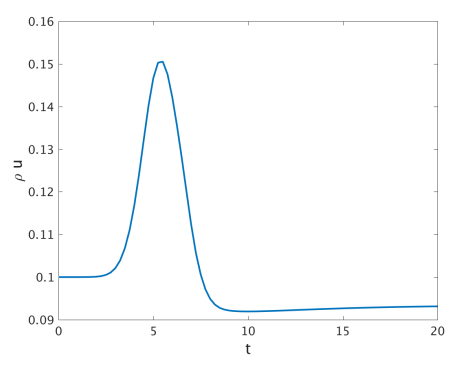

(b) $\boldsymbol{u}, 100 \%$ vortex

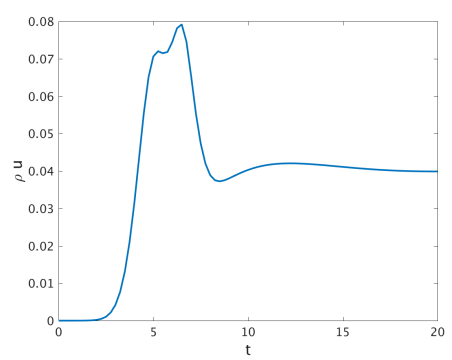

(e) $\boldsymbol{Q}, 100 \%$ vortex

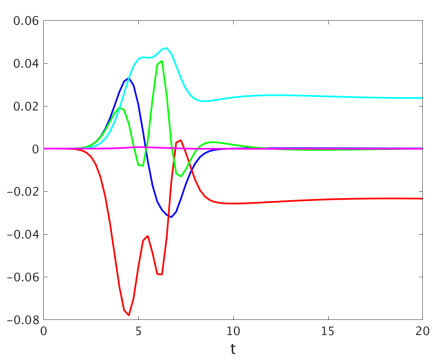

(h) $\boldsymbol{u}$-balance, $100 \%$ vortex

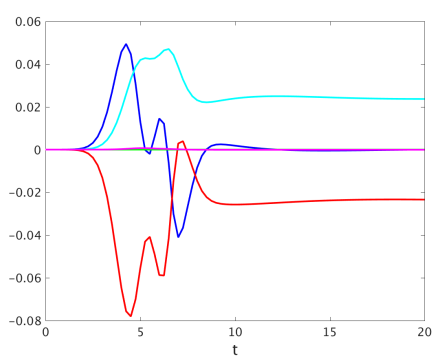

(k) $\boldsymbol{Q}$-balance, $100 \%$ vortex

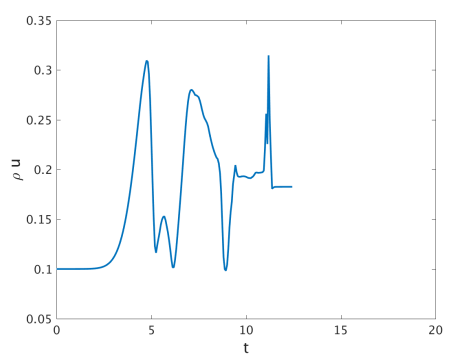

(c) $\boldsymbol{u}, 500 \%$ vortex

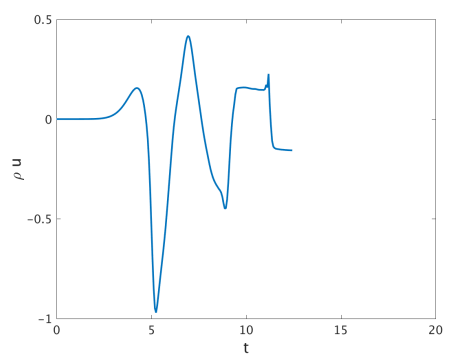

(f) $\boldsymbol{Q}, 500 \%$ vortex

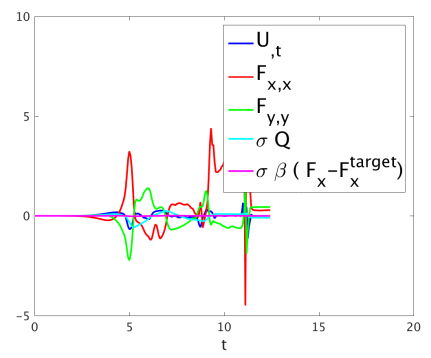

(i) $\boldsymbol{u}$-balance, $500 \%$ vortex

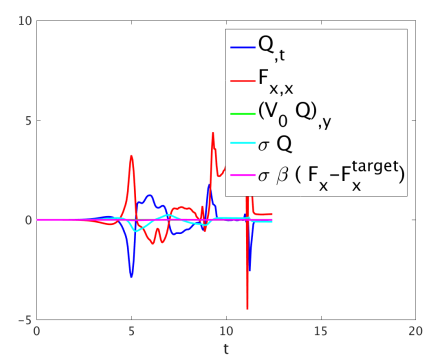

(1) $\boldsymbol{Q}$-balance, $500 \%$ vortex

Figure 17. Time history of $\mathrm{x}$-momentum state and PML variable within the PML domain for a convecting isentropic vortex. 


\section{References}

${ }^{1}$ Colonius, T., "Modeling Artificial Boundary Conditions for Compressible Flow," Annual Review of Fluid Mechanics, Vol. 36, 2004, pp. 315-345.

${ }^{2}$ Hedstrom, G. W., "Nonreflecting Boundary Conditions for Nonlinear Hyperbolic Systems," Journal of Computational Physics, Vol. 30, 1979, pp. 222-237.

${ }^{3}$ Thompson, K. W., "Time Dependent Boundary Conditions for Hyperbolic Systems," Journal of Computational Physics, Vol. 68, 1987, pp. 1-24.

${ }^{4}$ Giles, M. B., "Nonreflecting Boundary Conditions for Euler Equation Calculations," AIAA Journal, Vol. 28, No. 12, 2015/05/15 1990, pp. 2050-2058.

${ }^{5}$ Poinsot, T. J. and Lele, S. K., "Boundary Conditions for Direct Simulations of Compressible Viscous Flows," Journal of Computational Physics, Vol. 101, 1992, pp. 104-129.

${ }^{6}$ Lappas, T., Leonard, A., and Dimotakis, P. E., "Riemann Invariant Manifolds for the Multidimensional Euler Equations," SIAM Journal of Scientific Computing, Vol. 20, No. 4, 1999, pp. 1481-1512.

${ }^{7}$ Liu, Q. and Vasilyev, O. V., "Nonreflecting Boundary Conditions Based on Nonlinear Multidimensional Characteristics," International Journal for Numerical Methods in Fluids, Vol. 62, 2010, pp. 24-55.

${ }^{8}$ Tam, C. K. W. and Webb, J. C., "Dispersion-Relation-Preserving Finite-Difference Schemes for Computational Acoustics." Journal of Computational Physics, Vol. 107, 1993, pp. 262-281.

${ }^{9}$ Tam, C. K. W. and Dong, Z., "Radiation and Outflow Boundary Conditions for Direct Computation of Acoustic and Flow Disturbances in Nonuniform Mean Flow." Journal of Computational Acoustics, Vol. 4, 1996, pp. 175-201.

${ }^{10}$ Bogey, C. and Bailly, C., "Three-Dimensional Non-Reflective Boundary Conditions for Acoustic Simulations: Far Field Formulation and Validation Test Cases." Acta Acustica, Vol. 88, 2002, pp. 463-471.

${ }^{11}$ Israeli, M. and Orszag, S. A., "Approximation of Radiation Boundary Conditions," Journal of Computational Physics, Vol. 41, 1981, pp. 115-135.

${ }^{12}$ Berenger, J.-P., "A Perfectly Matched Layer for the Absorption of Electromagnetic Waves," Journal of Computational Physics, Vol. 114, No. 2, 1994, pp. $185-200$.

${ }^{13} \mathrm{Hu}$, F. Q., "On Absorbing Boundary Conditions for Linearized Euler Equations by a Perfectly Matched Layer," Journal of Computational Physics, Vol. 129, No. 1, 1996, pp. $201-219$.

${ }^{14} \mathrm{Hu}$, F. Q., "A Stable, Perfectly Matched Layer for Linearized Euler Equations in Unsplit Physical Variables," Journal of Computational Physics, Vol. 173, No. 2, 2001, pp. $455-480$.

${ }^{15} \mathrm{Hu}$, F. Q. and Atkins, H. L., "A Discrete Analysis of Non-Reflecting Boundary Conditions for Discontinuous Galerkin Method," AIAA Paper, 2003.

${ }^{16} \mathrm{Hu}$, F. Q., "A Perfectly Matched Layer Absorbing Boundary Condition for Linearized Euler Equations with a NonUniform Mean Flow," Journal of Computational Physics, Vol. 208, No. 2, 2005, pp. 469 - 492.

${ }^{17} \mathrm{Hu}$, F. Q., "Development of PML Absorbing Boundary Conditions for Computational Aeroacoustics: A Progress Review," Computers Fluids, Vol. 37, No. 4, 2008, pp. 336 - 348.

${ }^{18} \mathrm{Hu}, \mathrm{F} . \mathrm{Q}$., Li, X., and Lin, D., "Absorbing Boundary Conditions for Nonlinear Euler and Navier-Stokes Equations Based on the Perfectly Matched Layer Technique," Journal of Computational Physics, Vol. 227, No. 9, 2008, pp. 4398 - 4424.

${ }^{19} \mathrm{Lin}, \mathrm{D} ., \mathrm{Li}, \mathrm{X}$., and Hu, F. Q., "Absorbing Boundary Condition for Nonlinear Euler Equations in Primitive Variables Based on the Perfectly Matched Layer Technique," Computers Fluids, Vol. 40, No. 1, 2011, pp. 333 - 337.

${ }^{20}$ Parrish, S. A. and Hu, F. Q., "PML Absorbing Boundary Conditions for the Linearized and Nonlinear Euler Equations in the Case of Oblique Mean Flow," International Journal for Numerical Methods in Fluids, Vol. 60, No. 5, 2009 , pp. 565-589.

${ }^{21}$ Ismail, F. and Roe, P. L., "Affordable, Entropy-Consistent Euler Flux Functions II: Entropy Production at Shocks," Journal of Computational Physics, Vol. 228, 2009, pp. 5410-5436.

${ }^{22}$ Bassi, F. and Rebay, S., "GMRES Discontinuous Galerkin Solution of the Compressible Navier-Stokes Equations," Discontinuous Galerkin Methods: Theory, Computation and Application, Springer, Berlin, 2000, pp. 197-208.

${ }^{23}$ Diosady, L. T. and Murman, S. M., "Higher-Order Methods for Compressible Turbulent Flows Using Entropy Variables," AIAA Paper 2015-0294, 2015.

${ }^{24}$ Diosady, L. T. and Murman, S. M., "DNS of Flows over Periodic Hills Using a Discontinuous-Galerkin Spectral-Element Method," AIAA Paper 2014-2784, 2014.

${ }^{25}$ Garai, A., Diosady, L. T., Murman, S. M., and Madavan, N. K., "DNS of Flow in a Low-Pressure Turbine Cascade Using a Discontinuous-Galerkin Spectral-Element Method," ASME Turbo Expo Paper GT2015-42773, 2015.

26 "Second International Workshop on High-Order CFD Methods," http://www.dlr.de/as/desktopdefault.aspx/tabid8170/13999'read-35550/, 2013.

${ }^{27}$ Hillewaert, K., Carton de Wiart, C., Verheylewegen, G., and Arts, T., "Assessment of a High-Order Discontinuous Galerkin Method for the Direct Numerical Simulation of Transition at Low-Reynolds Number in the T106C High-Lift Low Pressure Turbine Cascade," ASME Turbo Expo Paper GT2014-26739, 2014.

${ }^{28}$ Roozeboom, N., Murman, S. M., Diosady, L., Burnside, N. J., and Ross, J. C., "Unsteady PSP Measurements on a Rectangular Cube," To be presented at AIAA SciTech, 2016. 